\title{
Gas-Liquid Two-Phase Flow Measurement Using Coriolis Flowmeters Incorporating Artificial Neural Network, Support Vector Machine, and Genetic Programming Algorithms
}

\author{
Lijuan Wang, Student Member, IEEE, Jinyu Liu, Yong Yan, Fellow, IEEE, Xue Wang, and Tao Wang
}

\begin{abstract}
Coriolis flowmeters are well established for the mass flow measurement of single-phase flow with high accuracy. In recent years, attempts have been made to apply Coriolis flowmeters to measure two-phase flow. This paper presents data driven models that are incorporated into Coriolis flowmeters to measure both the liquid mass flowrate and the gas volume fraction of a two-phase flow mixture. Experimental work was conducted on a purpose-built two-phase flow test rig on both horizontal and vertical pipelines for a liquid mass flowrate ranging from 700 to $14500 \mathrm{~kg} / \mathrm{h}$ and a gas volume fraction between $0 \%$ and $30 \%$. Artificial neural network (ANN), support vector machine (SVM), and genetic programming (GP) models are established through training with the experimental data. The performance of backpropagation-ANN (BP-ANN), radial basis function-ANN (RBF-ANN), SVM, and GP models is assessed and compared. Experimental results suggest that the SVM models are superior to the BP-ANN, RBF-ANN, and GP models for two-phase flow measurement in terms of robustness and accuracy. For liquid mass flowrate measurement with the SVM models, 93.49\% of the experimental data yield a relative error less than $\pm 1 \%$ on the horizontal pipeline, while $96.17 \%$ of the results are within $\pm 1 \%$ on the vertical installation. The SVM models predict the gas volume fraction with a relative error less than $\pm 10 \%$ for $93.10 \%$ and $94.25 \%$ of the test conditions on the horizontal and vertical installations, respectively.
\end{abstract}

Index Terms-Artificial neural network (ANN), Coriolis mass flowmeter, flow measurement, gas volume fraction, genetic programming (GP), support vector machine (SVM), two-phase flow.

\section{INTRODUCTION}

$\mathbf{G}$ AS-LIQUID two-phase flow is widely seen in oil and gas fields, chemical engineering, food processing, and other industrial processes. The accurate measurement of the

Manuscript received July 3, 2016; revised November 3, 2016; accepted November 7, 2016. Date of publication December 19, 2016; date of current version April 5, 2017. This work was supported by the UK CCS Research Centre which is a part of the RCUK Energy Programme founded by the EPSRC. The Associate Editor coordinating the review process was Dr. Domenico Grimaldi. (Corresponding author: Yong Yan.)

L. Wang, J. Liu, and Y. Yan are with the School of Engineering and Digital Arts, University of Kent, Canterbury CT2 7NT, U.K.

$\mathrm{X}$. Wang is with the School of Mathematics, Statistics and Actuarial Science, University of Kent, Canterbury CT2 7NF, U.K.

T. Wang is with KROHNE Ltd., Wellingborough NN8 6AE, U.K.

Color versions of one or more of the figures in this paper are available online at http://ieeexplore.iee.org.

Digital Object Identifier 10.1109/TIM.2016.2634630 flowrate of a two-phase mixture is challenging in industry. Significant research based on traditional flowmeters for twophase flow measurement has been conducted, such as Venturi, V-cone, turbine, vortex, and slotted orifice meters [1]-[3]. The determination of gas volume fraction of two-phase flow is crucial for the optimization of some industrial processes. Resistive sensors, capacitive sensors, electrical capacitance tomography, electrical resistance tomography, and microwave probes have been proposed for the phase fraction measurement of two-phase flow [4]-[6]. These techniques are often referred to as direct method, since the systems are designed to measure the desired two-phase flow characteristics directly. Due to the difficult nature of two-phase flow and complexity of the sensing systems, the applications of such direct two-phase flowmeters have achieved limited success in industry.

Indirect techniques based on traditional sensors incorporating soft-computing algorithms, such as artificial neural network (ANN), support vector machine (SVM), least-squares SVM, and extreme learning machine together with genetic algorithms or particle swarm optimization, have also been applied to two-phase or multiphase flow measurement or flow regime identification [7]-[10]. Coriolis flowmeters, as one of the most accurate single-phase mass flowmeters, have been successfully applied to a range of industrial applications. In recent years, many researchers have attempted to use Coriolis flowmeters for two-phase or multiphase flow measurement [11]. However, despite recent progress in sensor and transmitter technologies, improving the accuracy for mass flow metering of liquid with entrained gas still remains a challenge. A bubble effect model was proposed to study gasliquid two-phase flow for Coriolis flowmeters [12], but it cannot deal with positive errors in the mass flow measurement. Subsequently, Liu et al. [13] used a neural network to correct mass flow errors in a Coriolis mass flowmeter, which was based on a horizontal flow tube and the flow rate was limited to $1.5-3.6 \mathrm{~kg} / \mathrm{s}$. The multilayer perceptron and radial basis function (RBF) networks include four inputs, i.e., temperature, damping, density drop, and flowrate to estimate mass flow errors. Although most of the mass flow errors were reduced to within $\pm 2 \%$, the gas entrainment was not quantified and different installation conditions were not considered. A method based on fuzzy inference was proposed to correct the mass 
flow errors of a Coriolis flowmeter for the measurement of two-phase flow [14]. The fuzzy system accepts damping, drop in density, and apparent mass flowrate as inputs to generate corrected mass flowrate. Lari and Shabaninia [15] applied a neuro-fuzzy algorithm to the error correction of a Coriolis mass flowmeter for air-water two-phase flow measurement. However, the experimental data and the results were not explained in detail in [14] and [15]. Hou et al. [16] developed a digital Coriolis flow transmitter and tested a commercial Coriolis flowmeter. The measurement errors achieved under gas-liquid two-phase flow conditions were corrected using a feedforward neural network with two inputs-apparent liquid mass flowrate and apparent drop in density. Xing et al. [17] applied a Coriolis flowmeter in combination with an ultrasonic flowmeter to measure the individual mass flowrates of gasliquid two-phase flow under low liquid loading. The rootmean-square errors of gas and liquid mass flowrates were $3.09 \%$ and $12.78 \%$, respectively. Ma et al. [18] used a 25-mm bore Coriolis flowmeter together with SVM algorithms to measure the overall mass flowrate of oil-water two-phase flow and achieved relative errors within $\pm 1 \%$. The mass flowrate of individual phase was obtained with the maximum error of $\pm 8 \%$. However, it is known that the gas entrained in a liquid flow affects significantly the performance of Coriolis flowmeters, especially under different flow regimes [11]. Moreover, very little research has been undertaken to date to predict the gas volume fraction from the outputs of a Coriolis flowmeter.

Owning to the good reproducibility of the measurement errors of Coriolis flowmeters under two-phase flow conditions, data driven models, such as ANN, SVM, and genetic programming (GP), have the potential to correct the liquid mass flowrate and predict gas volume fraction. In this paper, experimental work was undertaken on a purpose-built 1-in $(25 \mathrm{~mm})$ bore air-water two-phase flow test rig. Coriolis flowmeters (KROHNE OPTIMASS 6400 S25) in conjunction with DP transducers were applied to obtain liquid mass flowrate and gas volume fraction on both the horizontal and vertical pipes. Parametric dependence along with input variable selection for the data driven models is investigated based on the partial mutual information (PMI) algorithm [19], [20]. Four data driven models based on backpropagation-ANN (BP-ANN), RBF-ANN, SVM, and GP, respectively, are established and validated through training and testing with the experimental data. The performances of the four models are evaluated and compared in terms of robustness and accuracy. The basic principle of BP-ANN modeling with some preliminary results was reported at the 2016 IEEE International Instrumentation and Measurement Technology Conference [21]. This paper presents in detail the principles, structures, training, and performance comparisons of the BP-ANN, RBF-ANN, SVM, and GP models.

\section{Methodology}

\section{A. Overall Measurement Strategy}

ANN, SVM, and GP are common data driven models for modeling a nonlinear system with multiple inputs and outputs [22]-[26]. These techniques learn from history data and give

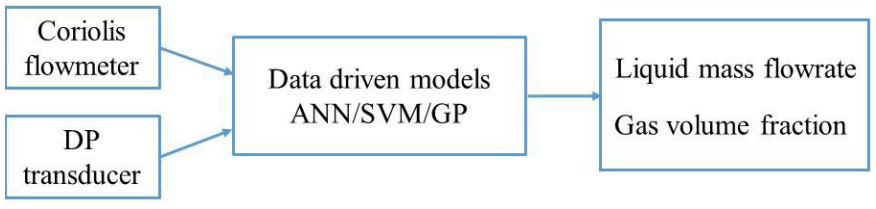

Fig. 1. Principle and structure of the measurement system.

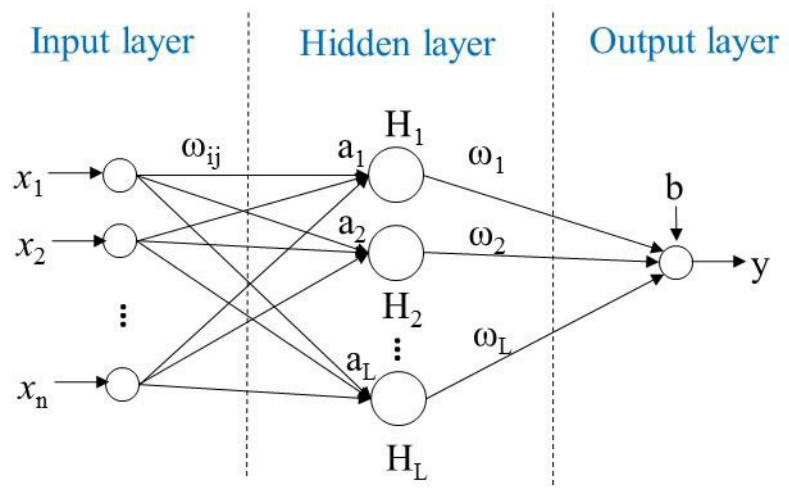

Fig. 2. Structure of a BP-ANN.

examples by constructing an input-output mapping in order to perform estimations of desired outputs. Fig. 1 shows the principle and structure of the measurement system. The data driven models accept variables from a Coriolis flowmeter and a DP transducer, while the output gives the corrected mass flowrate or predicted gas volume fraction. The analysis of parametric dependence and input variable selection for the data driven models based on the experimental data is presented in Section III-C. Since the volume of data is often limited in practice, it is appropriate to design a separate model for each desired output. The structure of each data driven model based on ANN, SVM, and GP will be explained in detail in Sections II.B-II.E.

\section{B. BP-ANN}

BP-ANN is a multilayer feedforward neural network trained with a BP learning algorithm, which is one of the most common neural networks. A BP-ANN consists of an input layer, one or more hidden layers, and an output layer. The hidden layer connects the input and output layers and represents their quantitative relationship. In general, a neural network with a single-hidden layer of sufficient neurons is able to represent any nonlinear problem. In consideration of the simplicity of the ANN structure, a single-hidden layer is chosen and investigated in this paper.

As shown in Fig. 2, $x=\left[x_{1}, x_{2}, \ldots, x_{n}\right]^{T}$ is an input sample and $y$ is the desired output. Assume $y$ is the linear output of the hidden neurons and a transfer function $f(x)$ is used on the neurons, the ANN is modeled as

$$
y_{\mathrm{BP}}=\sum_{j=1}^{L} \omega_{j} H_{j}+b=\sum_{j=1}^{L} \omega_{j} f\left(\sum_{i=1}^{n} \omega_{i j} x_{i}+a_{j}\right)+b
$$

where $n$ and $L$ are the numbers of input variables and hidden nodes. $\omega_{j}$ is the weight connecting the $j$ th hidden node and 


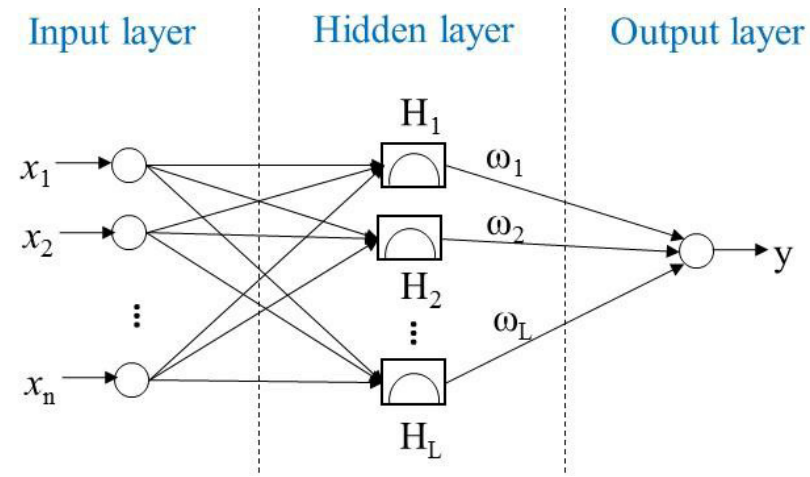

Fig. 3. Structure of an RBF-ANN.

the output node, and $\omega_{i j}$ is the weight connecting the $i$ th input node to the $j$ th hidden node. $a_{j}$ and $b$ are the biases on the $j$ th hidden node and the output node. In this paper, the hyperbolic tangent sigmoid function is used as a transfer function on hidden neurons and presented by

$$
f(x)=\frac{2}{1+e^{-2 x}}-1 .
$$

The learning algorithm is described as a procedure that consists of adjusting the weights and biases of a network, to minimize an error function between the network output and desired output for a given set of inputs. The BP algorithm has been widely applied to solve practical problems. However, the BP algorithm has the disadvantage of slow convergence and long training time. In addition, the success of the BP algorithm depends on the user-dependent parameters, such as initialization and structure of the ANN.

\section{RBF-ANN}

RBF-ANN has a fixed three layer structure (Fig. 3) and uses a type of RBF as an activation function to the hidden nodes. The output of the network is a linear combination of RBFs of the inputs and neuron parameters. The RBF measures the distance between the input vectors and the weight vectors and is typically taken to be the Gaussian function. Thus, the output of the network is given by

$$
y_{\mathrm{RBF}}=\sum_{j=1}^{L} \omega_{j} H_{j}=\sum_{j=1}^{L} \omega_{j} \exp \left(-\frac{1}{2 \sigma^{2}}\left\|x-C_{j}\right\|^{2}\right)
$$

where $C_{j}$ is the center vector for the $j$ th hidden node and determined by the K-means clustering method. $\left\|x-C_{j}\right\|$ is the Euclidean norm and $\sigma^{2}$ is the variance of the Gaussian function.

An RBF network with enough hidden nodes can approximate any continuous function with arbitrary precision. Moreover, as a local approximation network, the RBF neural network has the advantages of simple structure, less adjustive parameters, and fast training.

\section{SVM}

SVM was developed by Cortes and Vapnik [27] to solve the classification problem based on the statistic learning theory

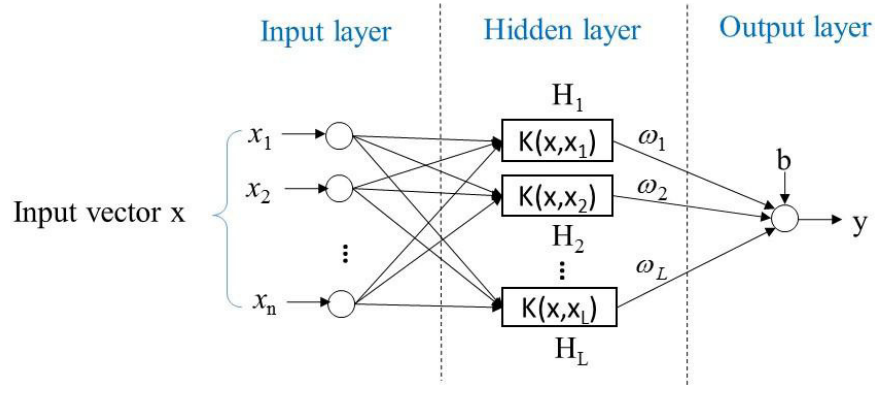

Fig. 4. Structure of an SVM.

and structural risk minimization. Then, this method has been extended to the domain of regression and prediction problems [28]. As shown in Fig. 4, the input vector $x$ is first mapped into an $L$-dimensional feature space using transfer functions, and then, a linear model is constructed in this feature space.

The linear model in the feature space is given by

$$
y=\omega x+b
$$

where $\omega=\left(\omega_{1}, \omega_{2}, \ldots, \omega_{\mathrm{L}}\right)$ is the weight vector and $b$ is the bias term.

Regression estimates can be obtained by minimizing the empirical risk on the training data. SVM regression performs a linear regression in the high-dimensional feature space using $\varepsilon$-insensitive loss and tends to reduce the model complexity by minimizing $\|\omega\|^{2}$. This can be described by introducing slack variables $\xi_{i}$ and $\xi_{i}^{\prime}(i=1,2, \ldots, m)$ to measure the deviation of training samples $\left(X^{*}, D\right)$ outside $\varepsilon$-insensitive zone. $X^{*}=\left(x^{1}, x^{2}, \ldots, x^{\mathrm{m}}\right)$ represents $m$ input vectors of training samples and $D=\left(d_{1}, d_{2}, \ldots, d_{\mathrm{m}}\right)$ is the corresponding desired output. Thus, the optimization problem can be formulated as

$$
\Phi=\min \frac{1}{2}\|\omega\|^{2}+C \sum_{i=1}^{m}\left(\xi_{i}+\xi_{i}^{\prime}\right)
$$

where $m$ is the number of training samples. $C$ is a positive constant as a regularization parameter that allows tuning the tradeoff between the flatness of the function and the tolerance of deviations larger than $\varepsilon$ (a constant).

Minimize the risk function of (5) subject to the following constraints:

$$
\begin{aligned}
d_{i}-y_{i} & \leq \varepsilon+\xi_{i} \\
y_{i}-d_{i} & \leq \varepsilon+\xi_{i}^{\prime} \\
\xi_{i} & \geq 0 \\
\xi_{i}^{\prime} & \geq 0 .
\end{aligned}
$$

Equation (4) can be transformed into a dual problem and solved by Lagrange functional

$$
y=\sum_{i=1}^{L}\left(\alpha_{i}-\alpha_{i}^{*}\right) \cdot K\left(x, x_{i}\right)+b
$$

where $\alpha_{i}$ and $\alpha_{i}^{*}$ are Lagrange multipliers and $K\left(x, x_{i}\right)$ is a kernel function. 


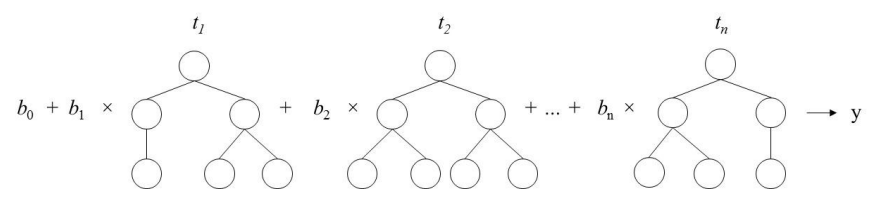

Fig. 5. Structure of a GP model.

There are some optional kernel functions for SVM, such as linear, polynomial, RBF, and sigmoid function. One of the most widely used kernel functions is the RBF. The final product of a training process in the SVM method can be presented by

$$
y_{\mathrm{SVM}}=\sum_{i=1}^{L}\left(\alpha_{i}-\alpha_{i}^{*}\right) \cdot \exp \left(-\frac{1}{2 \sigma^{2}}\left\|x-x_{i}\right\|^{2}\right)+b .
$$

\section{E. $G P$}

GP as an evolutionary computation technique is an extension of genetic algorithms and is widely applied to symbolic data mining (symbolic regression, classification, and optimization) [29]-[31]. Unlike the traditional regression analysis, GP-based symbolic regression automatically evolves both the structure and the parameters of the mathematical model from the available data. Meanwhile, it is superior to other machine learning techniques due to the ability to generate an empirical mathematical equation without assuming prior form of the existing relationships. In this paper, multigene symbolic regression is applied to establish a model for two-phase flow measurement. The structure of a multigene symbolic regression model is shown in Fig. 5.

The GP model can be regarded as a linear combination of lower order nonlinear transformations of the input variables. The output $y_{\mathrm{GP}}$ is defined as a vector output of $n$ trees modified by the bias term $b_{0}$ and scaling parameters $b_{1}, \ldots, b_{n}$

$$
y_{G P}=b_{0}+b_{1} t_{1}+\cdots+b_{n} t_{n}
$$

where $t_{i}(i=1, \ldots, n)$ is the $(m \times 1)$ vector of outputs from the $i$ th tree comprising a multigene individual.

The evolutionary process starts with initial population by creating individuals containing GP trees with different genes generated randomly. The evolutionary process continues with an evaluation of the fitness of the new population, two-point high-level crossover to acquire and delete genes, and low-level crossover on subtrees. Then, the created trees replace the parent trees or the unaltered individual in the next generation through mutation operators. The best program that appeared in any generation, the best-so-far solution, defines the output of the GP algorithm [30].

\section{EXPERIMENTAL Results AND Discussion}

\section{A. Test Rig and Experimental Conditions}

Fig. 6 shows the schematic of the two-phase flow test rig that was used in this paper. The measurement data obtained on this rig and subsequent conclusions drawn from the data

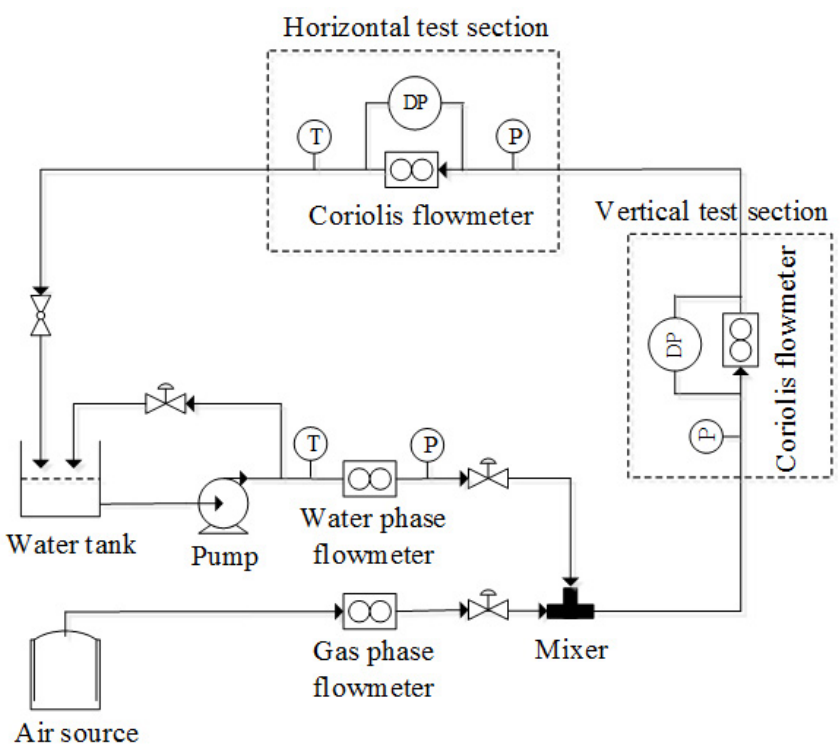

Fig. 6. Schematic of the two-phase flow test rig.

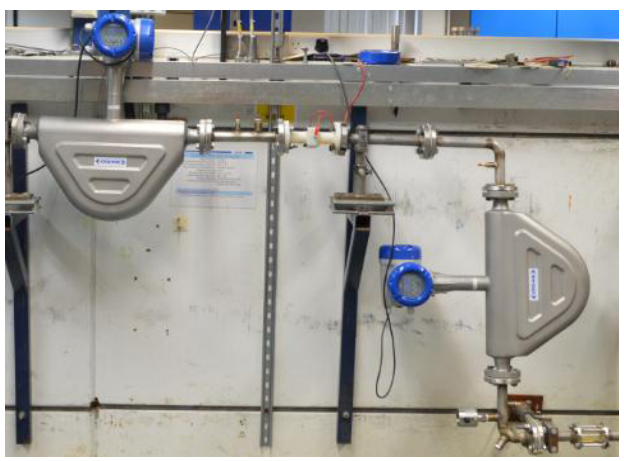

Fig. 7. Photograph of the test Coriolis flowmeters on 1-inch pipelines.

are expected to be transportable to other gas-liquid two-phase flow conditions. The gas flow is set to enter to the liquid flow through a bypass on the pipe. The liquid mass flowrate is controlled by adjusting the pump frequency from $15 \%$ to $80 \%$. The gas flowrate is varied by adjusting the opening of the valve in a gas flow controller. Two independent Coriolis flowmeters (KROHNE OPTIMASS 6400 S25 and Bronkhorst mini CORI-FLOW M15) were installed before the mixer to provide references for the individual mass flow rates of the liquid and gas phases, respectively. Both reference meters' measurement uncertainties under single-phase conditions were verified according to the manufacturer's technical specification. In the downstream, two additional Coriolis flowmeters (see Fig. 7) of the same type as the liquid reference meter were installed in the vertical and horizontal test sections, respectively. These are the meters under test to assess the performance of ANN, SVM, and GP models under twophase flow conditions. In view of the effects of gravity and buoyancy on two-phase fluid, both the horizontal and vertical installations of the meters are considered. A DP transducer was used to record the DP value across each flowmeter under test. 

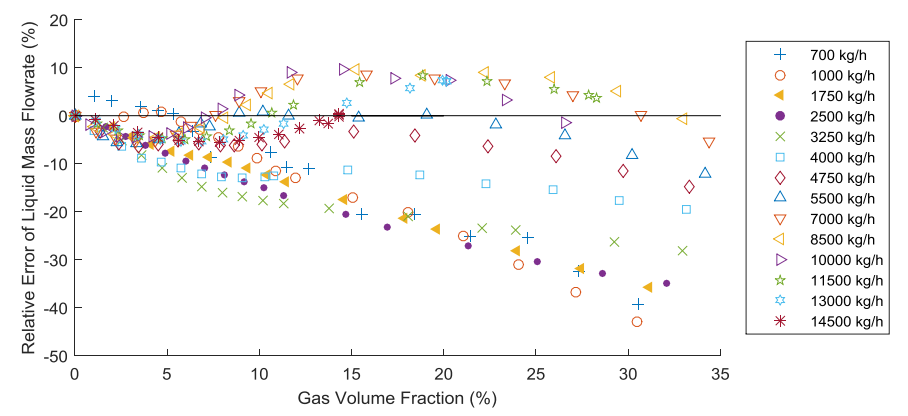

(a)

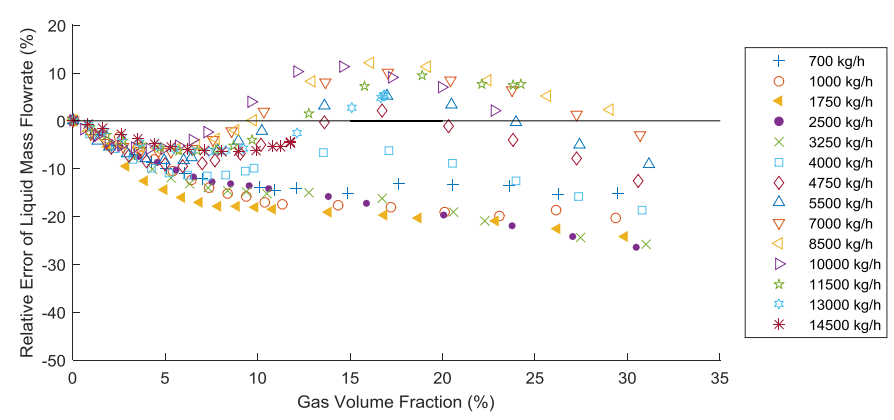

(b)

Fig. 8. Original errors of the liquid mass flowrate from Test I. (a) Horizontal pipeline. (b) Vertical pipeline.

The data logging frequencies, as set in the data loggers for the mass flowrate, density, damping, and DP, are 50, 10, 2, and $20 \mathrm{~Hz}$, respectably. Each parameter was logged over a period of $100 \mathrm{~s}$ with a time averaged value generated under each experimental condition. Gas volume fraction $\alpha$ is defined and calculated as follows:

$$
\alpha=\frac{q_{v, g}}{q_{v, l}+q_{v, g}} \times 100 \%
$$

where $q_{v, g}$ and $q_{v, l}$ are the calculated volume flowrates of gas and liquid phases from the reference flow meters and the temperature and pressure in the upstream of the horizontal test meter.

Density drop is determined from the density of the liquid flow $\left(\rho_{l}\right)$ and the apparent density $(\rho)$ from the Coriolis flowmeter under test

$$
d=\frac{\rho_{l}-\rho}{\rho_{l}} \times 100 \% .
$$

Two series of experimental tests, Tests I and II, were conducted for the liquid mass flow rate ranging from 700 to $14500 \mathrm{~kg} / \mathrm{h}$ and gas volume fraction from $0 \%$ to $30 \%$. The fluid temperature during the tests was around $20{ }^{\circ} \mathrm{C}$. For the purpose of ANN training, 237 data sets were collected from Tests I, while 24 data sets recorded from Tests II for testing the performance of the data driven models.

\section{B. Analysis of Original Errors}

The typical original mass flow errors of the Coriolis flowmeters in Test I are shown in Fig. 8. The Coriolis flowmeter on the vertical section gives negative errors at flowrates below $4000 \mathrm{~kg} / \mathrm{h}$. At a higher flowrate $(>5500 \mathrm{~kg} / \mathrm{h})$, the mass flow

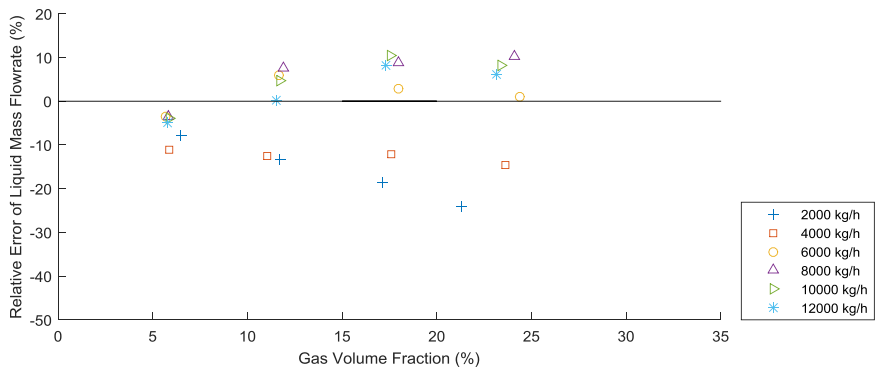

(a)

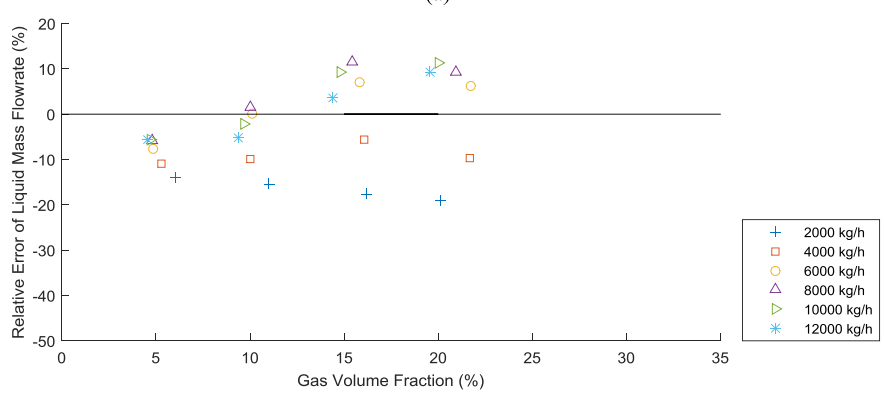

(b)

Fig. 9. Original errors of the liquid mass flowrate from Test II. (a) Horizontal pipeline. (b) Vertical pipeline.

errors become positive and crossing the zero line and then return to negative errors again along with increasing entrained gas. This is believed to be due to the flow regime effects on the fluid-tube coupling system at different flowrates. At a lower flowrate $(<2000 \mathrm{~kg} / \mathrm{h})$, the flow was nearly slug flow as observed during the test, while the flow regime became gradually dispersed bubbly flow as the flowrate and entrained gas increase. For the Coriolis flowmeter on the horizontal pipeline, the range of mass flow errors is different from that on the vertical pipeline most likely due to the effects of gravity and buoyancy on the flow regime. Positive errors occur at the mass flowrates of 700 and $1000 \mathrm{~kg} / \mathrm{h}$ when the gas volume fraction below 6\%. By comparing the mass flow errors at the same flowrate in Figs. 8 and 9, the errors are generally reproducible for the same installation and thanks to the new-generation flow transmitter [32]. For the test data set, Test II includes some experimental data that were collected at different flowrates from those in Test I. The new conditions as in Test II that were conducted on a different day and obtained under different flowrate from Test I are useful to assess the models' generalization capability and reproducibility.

Fig. 10 shows the distribution of the relative errors of the measured liquid mass flowrate on both the horizontal and vertical pipelines. Each color (blue or green) in the figure represents training or test data sets, respectively. The Coriolis flowmeter on the horizontal pipeline yields the liquid mass flowrate with a relative error between $-41 \%$ and $9 \%$, while the meter on the vertical pipeline gives an error from $-25 \%$ to $11 \%$. The difference in errors between the vertical and horizontal installations is due to the fact that the bubbles in a vertical flow are distributed evenly in the pipe cross section due to the effect of gravity, resulting in less interruption on the tube vibration inside the Coriolis flowmeter and hence different errors. 


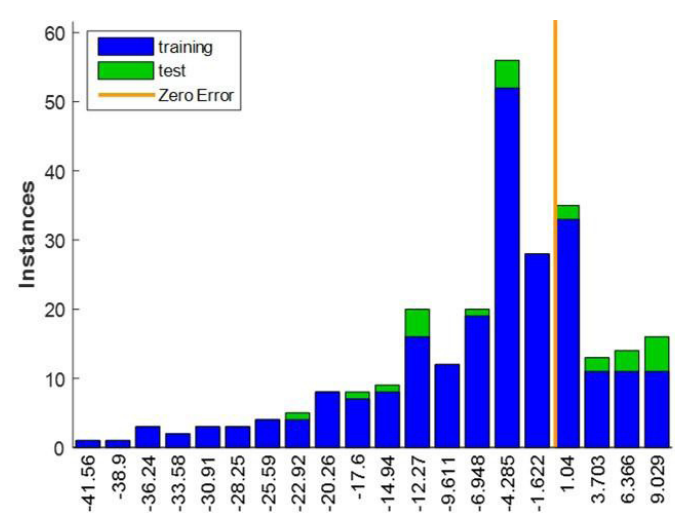

Relative Error (\%)

(a)

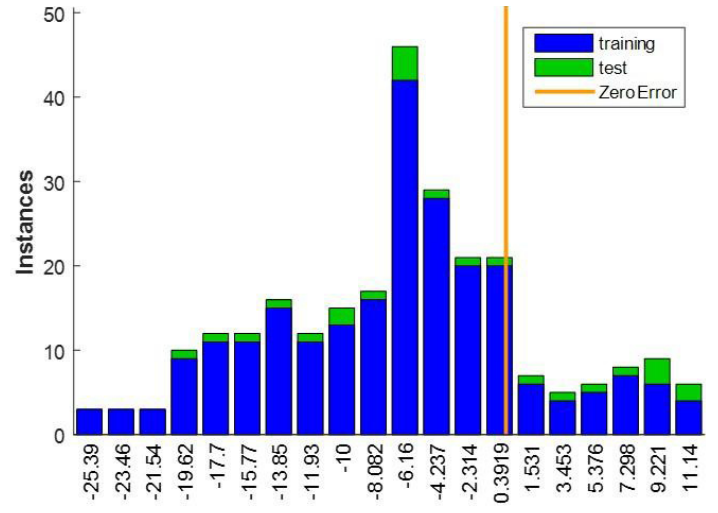

Relative Error (\%)

(b)

Fig. 10. Relative error histogram of the measured liquid mass flowrate. (a) Horizontal pipeline. (b) Vertical pipeline.

\section{Analysis of Parametric Dependence}

There are three important parameters from a Coriolis flowmeter, including observed density drop, apparent mass flowrate, and damping. The DP value from the DP transducer is also included as a potential input variable in this paper. The apparent mass flowrate from a Coriolis flowmeter and the DP value across the meter correlate strongly with the liquid mass flowrate under two-phase conditions. In addition, when gas entrains in the liquid flow, a rapid rise in damping occurs for the fluid-conveying tube and the mixture density also deviates from the liquid density. This physical background for the fluidtube coupling system determines that these four input variables are more important than other variables. There exist strong nonlinearities between the outputs of a Coriolis flowmeter and the flowrate being measured under two-phase flow conditions, as observed by other researchers [12], [13]. Such nonlinearities are also shown in Fig. 8.

In order to investigate the parametric dependence of individual input parameters and the combined effect of multiple parameters on the output of a data model, PMI is utilized to measure the partial dependence between a potential input variable and the output, conditional on any inputs that have already been selected. The variable with the highest PMI score is added to the input set, if the Akaike information criterion (AIC) value decreases as a result from the inclusion of this variable. The detailed definitions of PMI and AIC are available in [19] and [20]. Suppose variables $x_{1}, x_{2}, x_{3}$, and $x_{4}$ represent observed density drop, apparent mass flowrate, damping, and DP, respectively, the variable selection procedures for the models for correcting the liquid mass flowrate and predicting the gas volume fraction are summarized in Tables I and II. $\mathrm{H}-\mathrm{L}$ and V-L represent the models established for the horizontal and vertical pipelines, respectively, to correct the liquid mass flowrate, while H-G and V-G stand for the models for the horizontal and vertical pipelines to predict the gas volume fraction, respectively. The selection sequence also represents the sensitivity level of each variable to the desired output. For the liquid mass flowrate, $x_{2}$ (apparent mass flowrate) has more significant effect on the liquid mass flowrate.
TABLE I

VARiable Selection Procedures for Models H-L AND V-L

\begin{tabular}{|c|c|c|c|c|c|c|}
\hline \multirow{2}{*}{ Step } & \multicolumn{3}{|c|}{ Model H-L } & \multicolumn{3}{c|}{ Model V-L } \\
\cline { 2 - 7 } & Variable & AIC & $\mathrm{R}^{2}$ & Variable & AIC & $\mathrm{R}^{2}$ \\
\hline I & $x_{2}$ & -1030 & 0.9795 & $x_{2}$ & -1027 & 0.9793 \\
\hline II & $x_{3}$ & -971 & 0.9757 & $x_{4}$ & -1032 & 0.9797 \\
\hline III & $x_{4}$ & -1040 & 0.9814 & $x_{3}$ & -1085 & 0.9842 \\
\hline IV & $x_{1}$ & -1161 & 0.9886 & $x_{1}$ & -1200 & 0.9901 \\
\hline
\end{tabular}

TABLE II

VAriable Selection Procedures for Models H-G AND V-G

\begin{tabular}{|c|c|c|c|c|c|c|}
\hline \multirow{2}{*}{ Step } & \multicolumn{3}{|c|}{ Model H-G } & \multicolumn{3}{c|}{ Model V-G } \\
\cline { 2 - 7 } & Variable & AIC & $\mathrm{R}^{2}$ & Variable & AIC & $\mathrm{R}^{2}$ \\
\hline I & $x_{1}$ & -669.3 & 0.9210 & $x_{1}$ & -485.8 & 0.8430 \\
\hline II & $x_{4}$ & -743.3 & 0.9434 & $x_{4}$ & -668.1 & 0.9248 \\
\hline III & $x_{2}$ & -745.9 & 0.9456 & $x_{2}$ & -691.1 & 0.9334 \\
\hline IV & $x_{3}$ & -727.4 & 0.9415 & $x_{3}$ & -669.2 & 0.9272 \\
\hline
\end{tabular}

The coefficient of determination, $R^{2}$, indicates the goodness of fit. A combination of the four variables gives the highest $R^{2}$, which illustrates that the combined effect of the variables is more significant than that of an individual variable on the output. For predicting the gas volume fraction, $x_{1}$ (observed density drop), plays a more important part than other variables. Variable $x_{3}$ (damping) is not used in models H-G and V-G, since the AIC value becomes increasing and $R^{2}$ is reducing with the inclusion of $x_{3}$. As a result of these variable selection procedures, the models for correcting the liquid mass flowrate accept the four input variables (observed density drop, apparent mass flowrate, damping, and DP) and three variables (observed density drop, apparent mass flowrate, and DP) are taken as the inputs to the models for predicting the gas volume fraction.

\section{Performance of the BP-ANN}

The BP-ANN model is established through training with data set I and tested with data set II. For each installation 


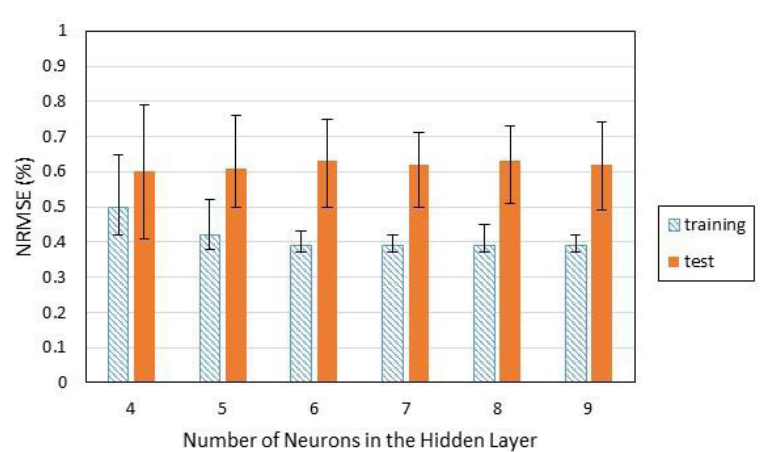

(a)

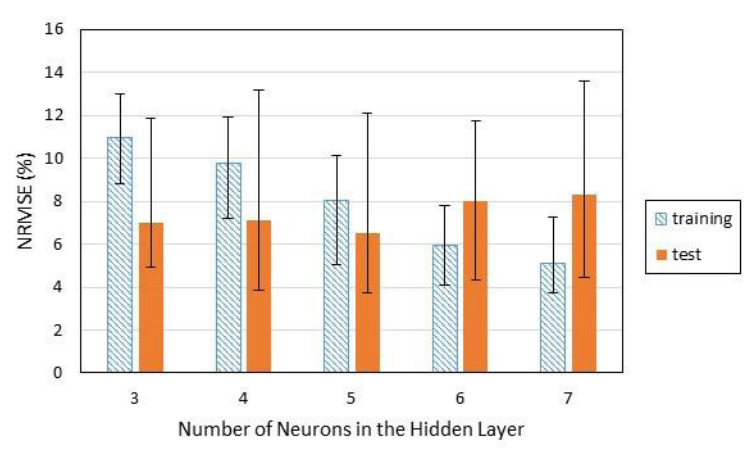

(c)

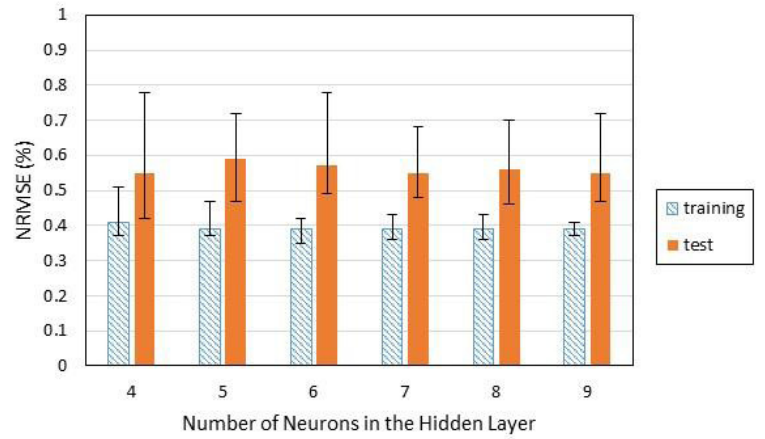

(b)

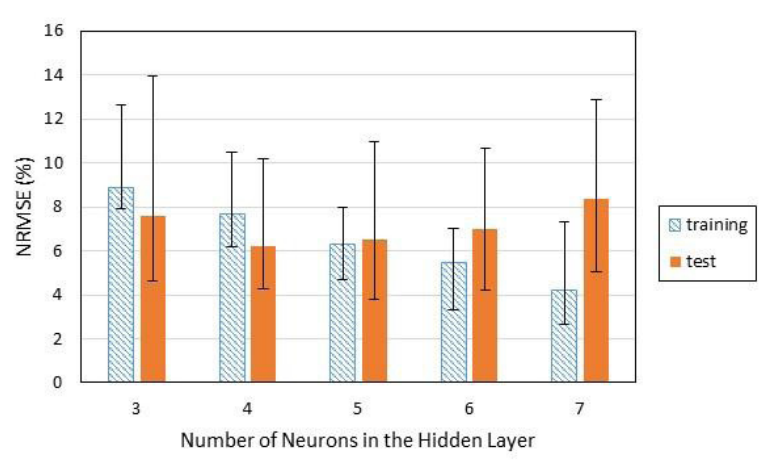

(d)

Fig. 11. Performance of BP-ANNs with differenct numbers of neurons in the hidden layer. (a) BP-ANN: H-L. (b) BP-ANN: V-L. (c) BP-ANN: H-G. (d) BP-ANN: V-G.

condition, a separate model is established for the correction of the measured liquid mass flowrate and the prediction of gas volume fraction. The inputs of the BP-ANN for liquid mass flowrate correction include four variables, i.e., observed density drop, apparent mass flowrate, damping, and DP. The inputs of the BP-ANN for gas volume fraction prediction include observed density drop, apparent mass flowrate, and DP. The number of neurons $(L)$ in the hidden layer is determined using (15) and (16), as proposed in [33]

$$
\begin{aligned}
& L \leq 2 n+1 \\
& L \leq \frac{m}{n+1}
\end{aligned}
$$

where $n$ and $m$ are the numbers of input variables and training samples, respectively. However, (15) and (16) give only the range of $L$ for BP-ANN models. The exact $L$ for a model can be selected by a trial-and-error method to compromise between minimizing errors and achieving good generalization capability. The output layer has one neuron for each model, since there is only one output variable.

The BP-ANN transfer function between the input and hidden layers is hyperbolic tangent sigmoid transfer function. The pure linear function is taken as the transfer function connecting the hidden layer to the output layer. The training function is Bayesian regularization, while the learning function is gradient descent with momentum weight and bias learning function. Training stops when the maximum number of epochs is reached or the performance is minimized to the goal. In this paper, normalized root-mean-square error (NRMSE) is used to assess the performance of a data driven model, which is defined as

$$
N R M S E=\frac{1}{\bar{y}} \sqrt{\frac{1}{m} \sum_{i=1}^{m}\left(y_{i}-\hat{y}_{i}\right)^{2}}
$$

where $y_{i}$ is the reference mass flow rate of the liquid phase or gas volume fraction, $\bar{y}$ is the mean of $y_{i}, \hat{y}_{i}$ is the corrected mass flow rate or predicted gas volume fraction from the data driven model accordingly, and $m$ is the number of samples used.

As the weights and biases between the neurons are initialized randomly, a different BP-ANN is obtained for each training, resulting in different performances. A preliminary study of averaging NRMSE of more than 200 BP-ANNs did not show any noticeable difference. Therefore, in order to minimize the effect of random initialization of an ANN, the average NRMSE of 200 BP-ANNs with the same structure is calculated to assess the effect of the hidden neurons on the performance of the ANN.

For the models for liquid mass flowrate correction, the number of neurons in the hidden layer is set from 4 to 9 as per (15) and (16). The NRMSE values of the BP-ANNs are summarized in Fig. 11. The error bars indicate the maximum and minimum errors of $200 \mathrm{BP}-\mathrm{ANNs}$ for the same structure. In view of the errors on both training and test datasets, the BP-ANN with seven neurons in the hidden layer performs 


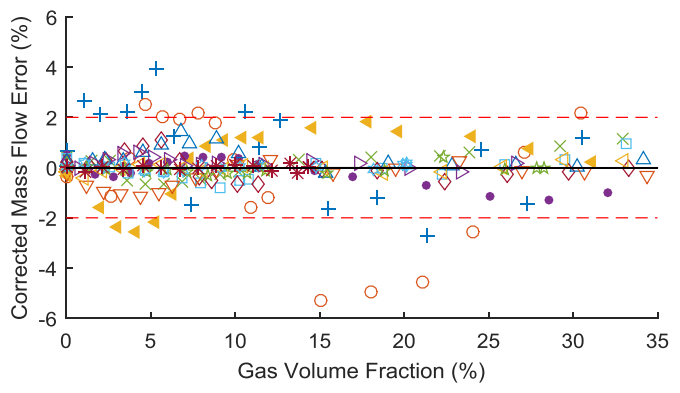

(a)

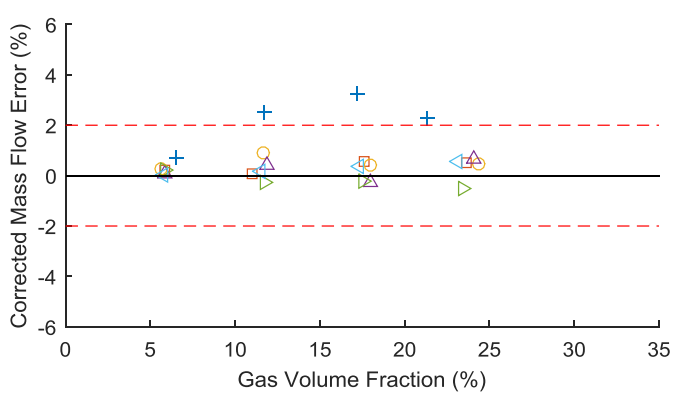

(b)

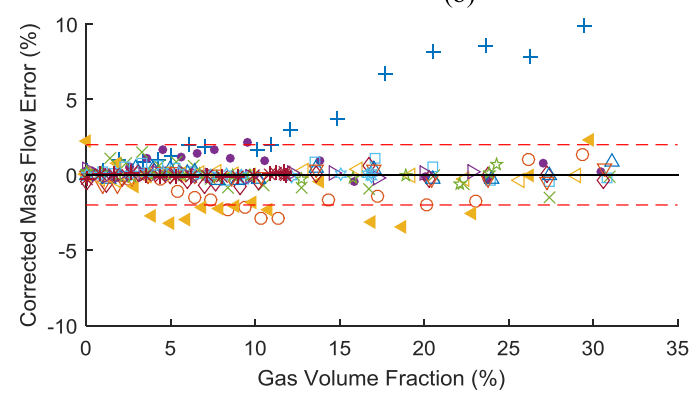

(c)

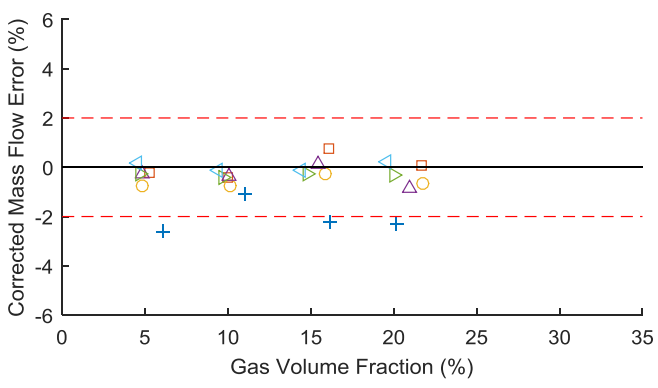

(d)

Fig. 12. Errors of the corrected liquid mass flowrate from the trained BP-ANNs. (a) Errors of the corrected mass flowrate on the horizontal pipeline with training data set. (b) Errors of the corrected mass flowrate on the horizontal pipeline with test data set. (c) Errors of the corrected mass flowrate on the vertical pipeline with training data set. (d) Errors of the corrected mass flowrate on the vertical pipeline with test data set.

better than other structures under both the horizontal and vertical conditions. The BP-ANN used for gas volume fraction prediction has lower NRMSE when the number of the hidden neurons is 6 .

Once the structure of a BP-ANN is determined, the trained neural network that has the minimum error with the test data set is selected. Fig. 12 shows the errors of the corrected

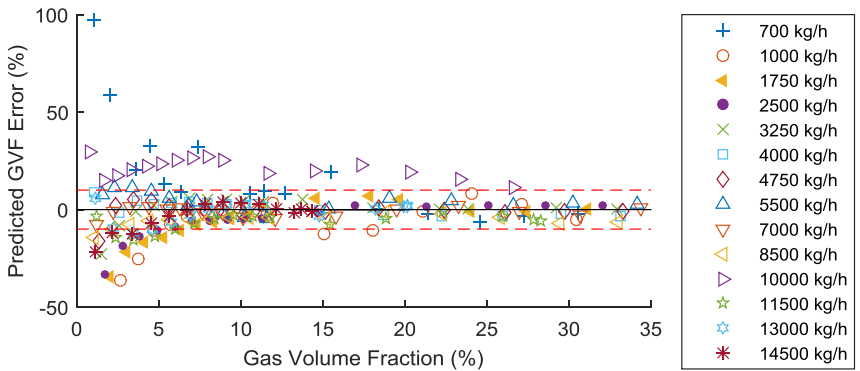

(a)

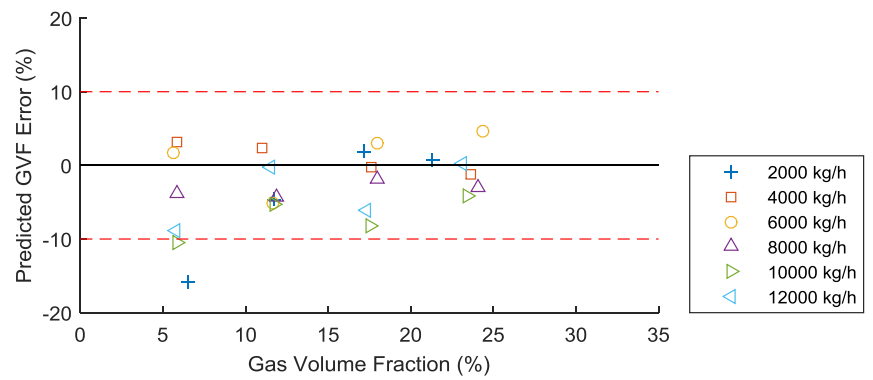

(b)

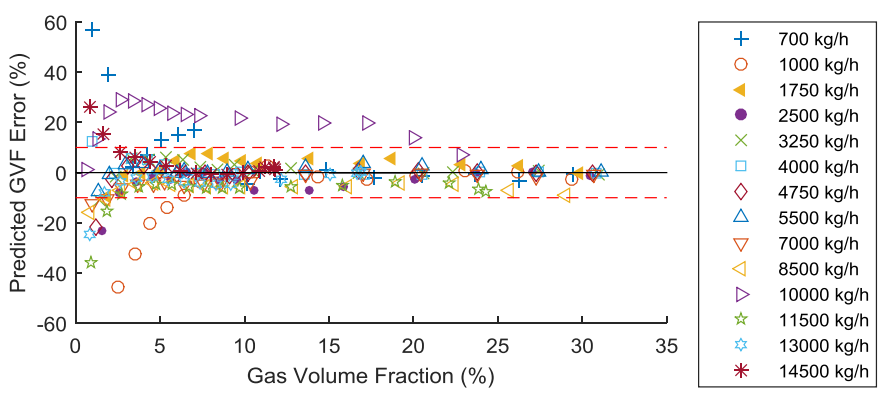

(c)

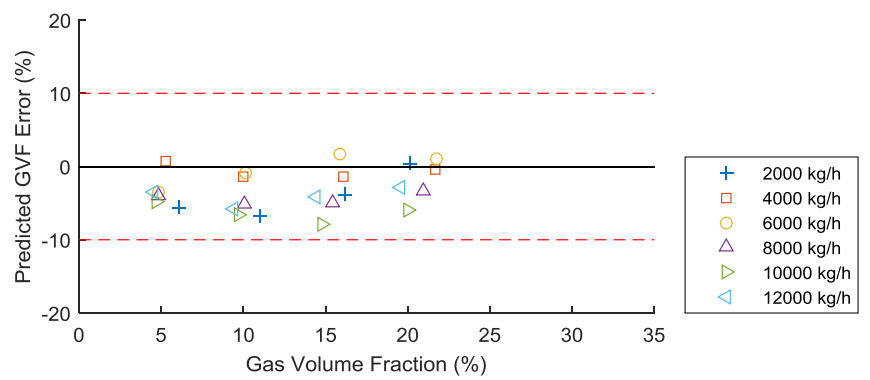

(d)

Fig. 13. Error of the predicted gas volume fraction from the trained BP-ANNs. (a) Errors of the predicted gas volume fraction on the horizontal pipeline with training data set. (b) Errors of the predicted gas volume fraction on the horizontal pipeline with test data set. (c) Errors of the predicted gas volume fraction on the vertical pipeline with training data set. (d) Errors of the predicted gas volume fraction on the vertical pipeline with test data set.

liquid mass flowrate from the BP-ANNs. For the horizontal and vertical pipelines, the relative errors are mostly less than $\pm 2 \%$ (the red dashed lines in Fig. 12) with the training data set except some larger errors at the low flowrates of 700 and $1000 \mathrm{~kg} / \mathrm{h}$. This is very likely due to larger bubbles or slugs appearing in the flow tubes under low flowrate, which affects the Coriolis flowmeter behaving differently from 


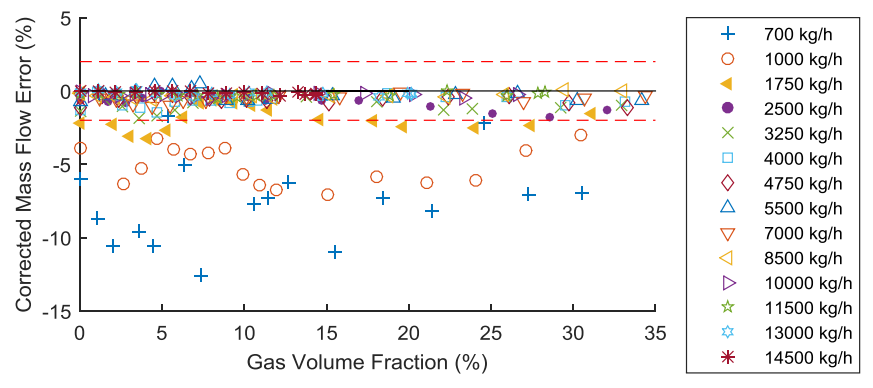

(a)

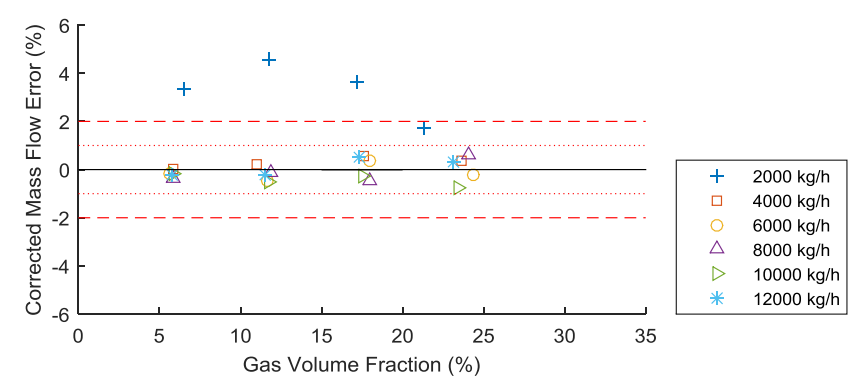

(b)

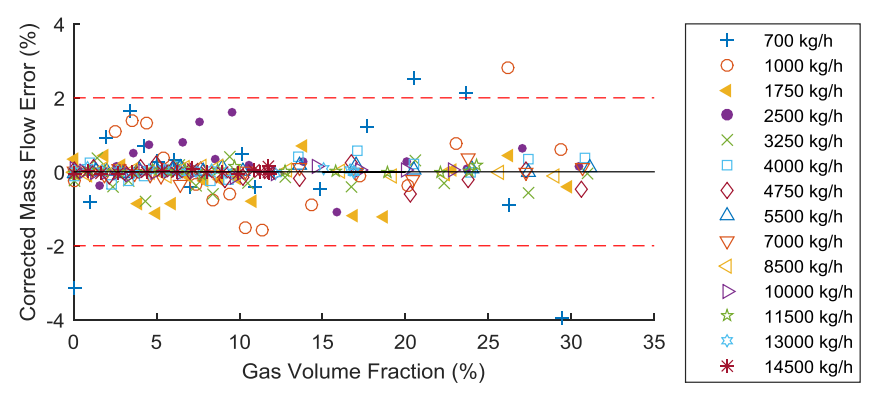

(c)

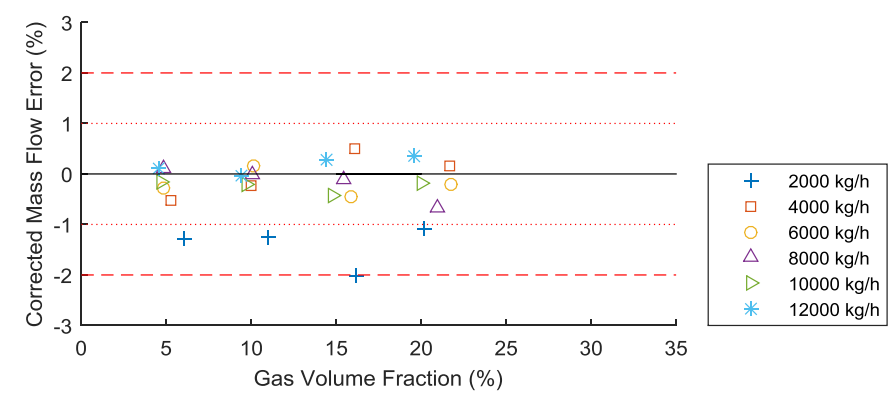

(d)

Fig. 14. Errors of the corrected liquid mass flowrate from the RBF-ANNs. (a) Errors of the corrected mass flowrate on the horizontal pipeline with training data set. (b) Errors of the corrected mass flowrate on the horizontal pipeline with test data set. (c) Errors of the corrected mass flowrate on the vertical pipeline with training data set. (d) Errors of the corrected mass flowrate on the vertical pipeline with test data set.

smaller bubbles. The trained BP-ANN has relatively larger errors at low flowrates and hence results in unsatisfactory performance with the test data set under the same experimental conditions.

Since the gas volume fraction under the experimental conditions ranges from $0 \%$ to $30 \%$ and the intrinsic complexity of two-phase flow, the relative errors of the predicted gas volume fraction from the BP-ANNs are quite large when the gas

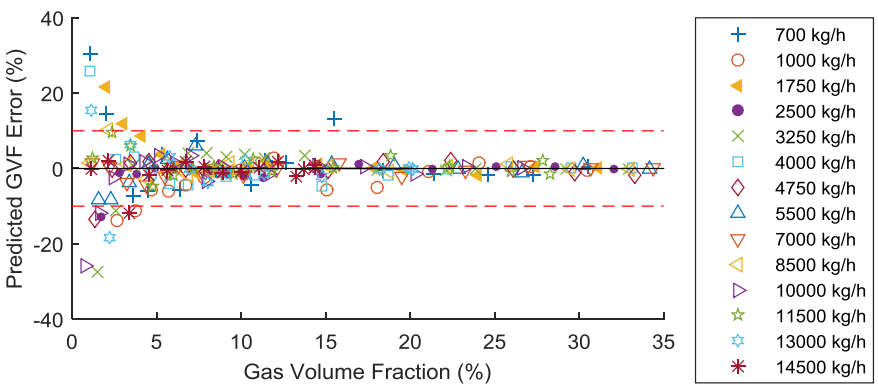

(a)
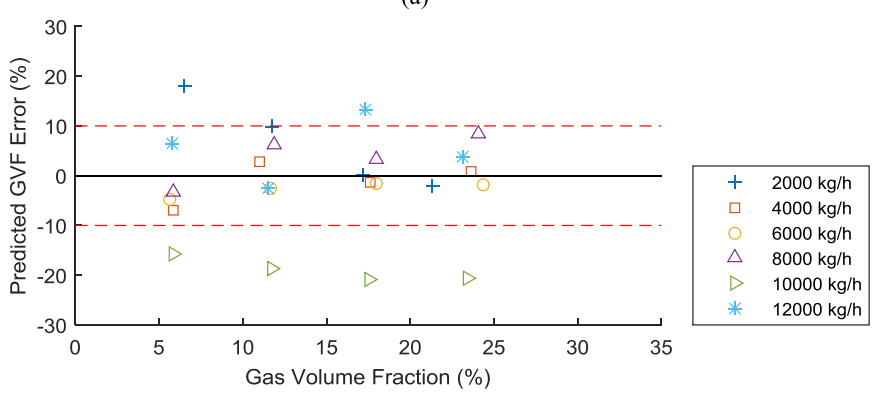

(b)

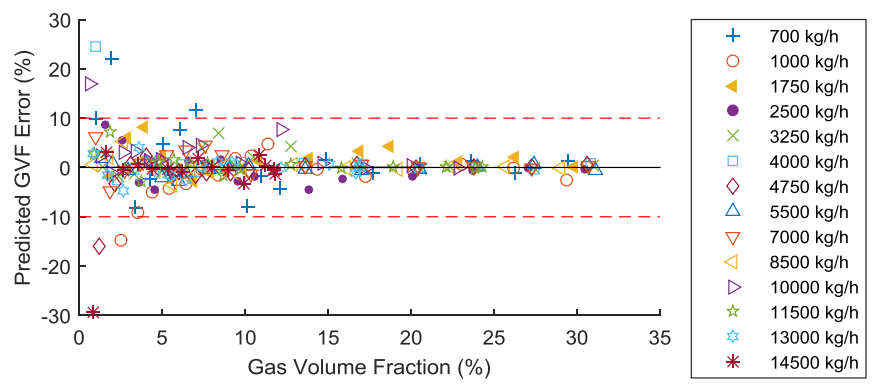

(c)

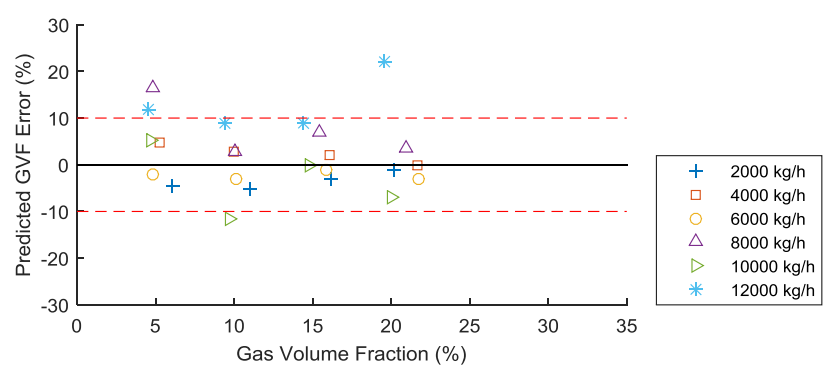

(d)

Fig. 15. Errors of the predicted gas volume fraction from the RBF-ANNs. (a) Errors of the predicted gas volume fraction on the horizontal pipeline with training data set. (b) Errors of the predicted gas volume fraction on the horizontal pipeline with test data set. (c) Errors of the predicted gas volume fraction on the vertical pipeline with training data set. (d) Errors of the predicted gas volume fraction on the vertical pipeline with test data set.

volume fraction is below 5\%. As the entrained gas increases, the errors from the training data set are mostly within $\pm 10 \%$ (the red dashed lines in Fig. 13). For the test data set, however, all the errors are less than $\pm 10 \%$ on the vertical pipeline, even under the low flowrate conditions.

\section{E. Performance of the RBF-ANN}

Fig. 14 shows the relative errors of the corrected liquid mass flowrate from the RBF-ANNs. In order to achieve more 


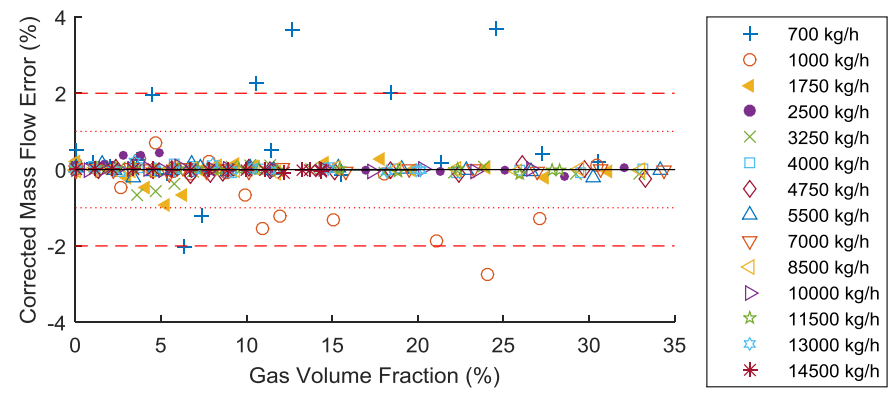

(a)

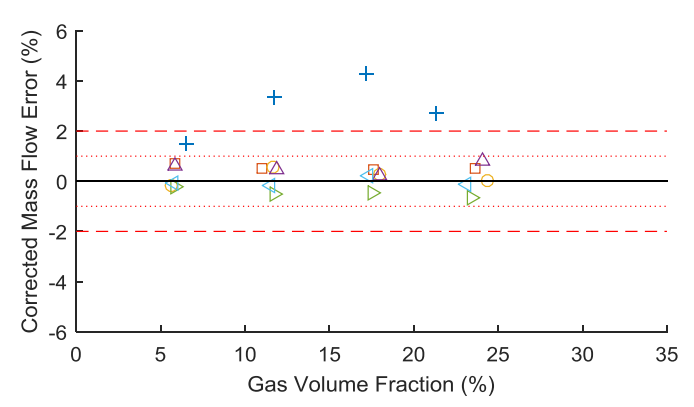

(b)

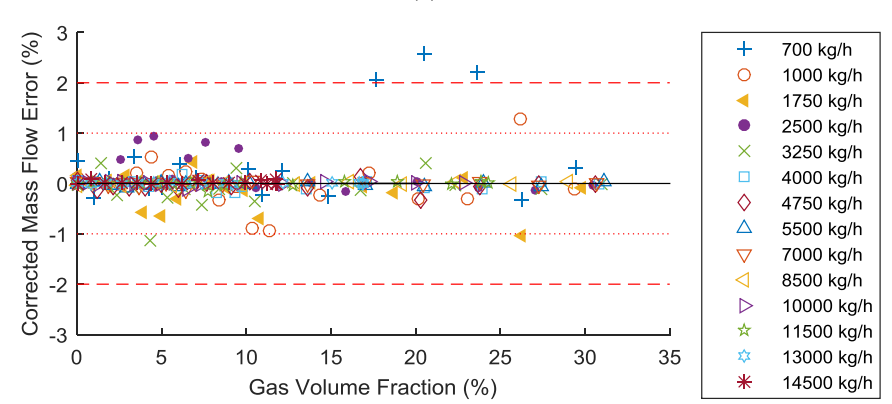

(c)

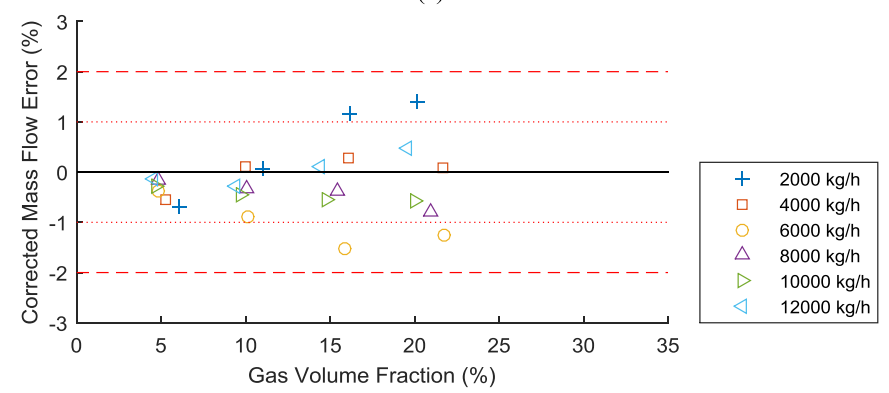

(d)

Fig. 16. Errors of the corrected liquid mass flowrate error from the SVMs. (a) Errors of the corrected mass flowrate on the horizontal pipeline with training data set. (b) Errors of the corrected mass flowrate on the horizontal pipeline with test data set. (c) Errors of the corrected mass flowrate on the vertical pipeline with training data set. (d) Errors of the corrected mass flowrate on the vertical pipeline with test data set.

accurate results with the test data set, the RBF-ANN on the horizontal pipeline disregards the errors at lower flowrates $(<2000 \mathrm{~kg} / \mathrm{h})$ and the network is trained to well fit higher flowrates $(>4000 \mathrm{~kg} / \mathrm{h})$. Consequently, the errors at higher flowrates with the training data set, and the errors with the test data set are reduced to $\pm 1 \%$. Due to the insignificant

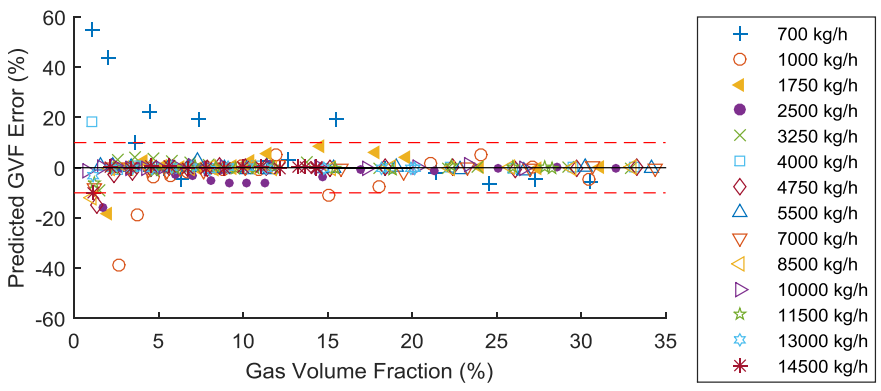

(a)
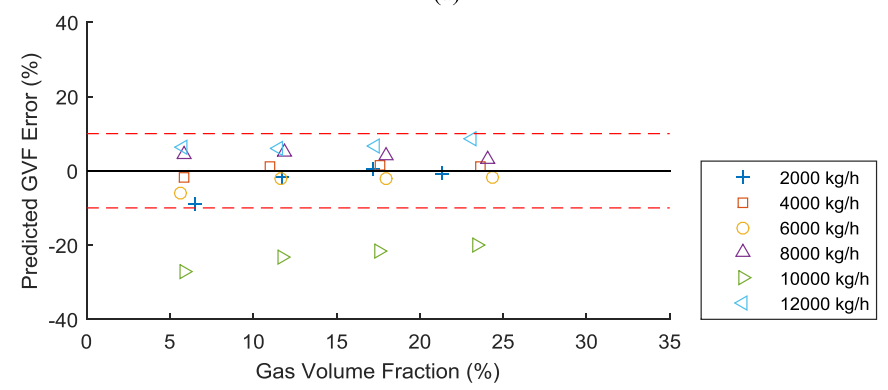

(b)

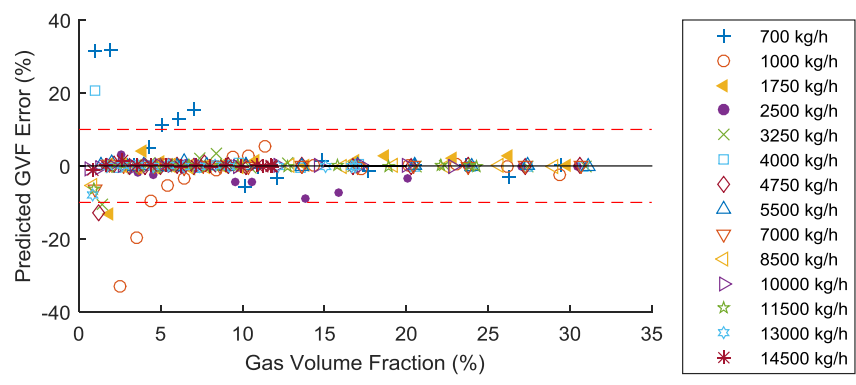

(c)

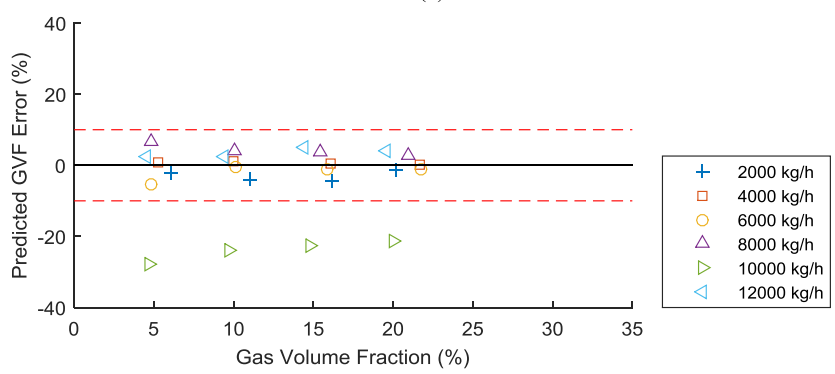

(d)

Fig. 17. Errors of the predicted gas volume fraction from the SVMs. (a) Errors of the predicted gas volume fraction on the horizontal pipeline with training data set. (b) Errors of the predicted gas volume fraction on the horizontal pipeline with test data set. (c) Errors of the predicted gas volume fraction on the vertical pipeline with training data set. (d) Errors of the predicted gas volume fraction on the vertical pipeline with test data set.

difference in the original errors between the lower and higher flowrates on the vertical pipeline, the RBF-ANN yields errors between $\pm 2 \%$ with the training data set and $\pm 1 \%$ with the test data set.

As shown in Fig. 15, the RBF-ANN for gas volume fraction prediction outperforms significantly the BP-ANN, particularly under the low entrained gas. When the gas volume fraction is below $5 \%$, the maximum relative errors from RBF-ANNs on 

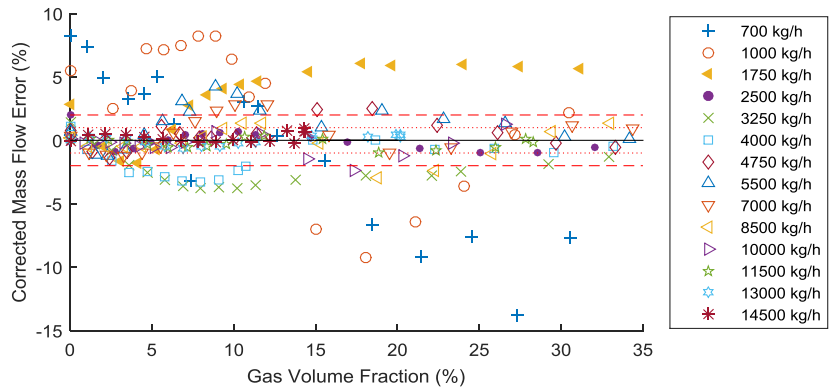

(a)

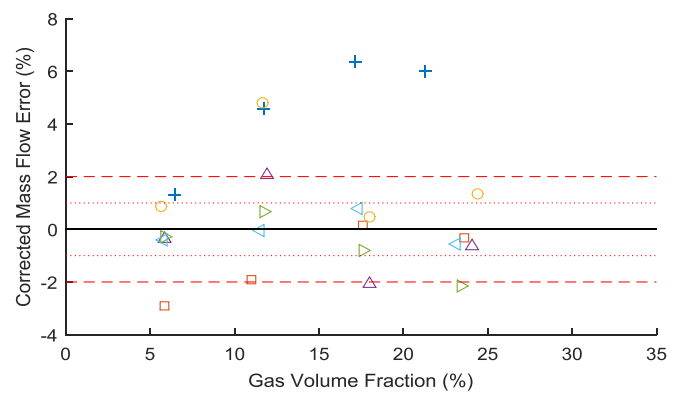

(b)
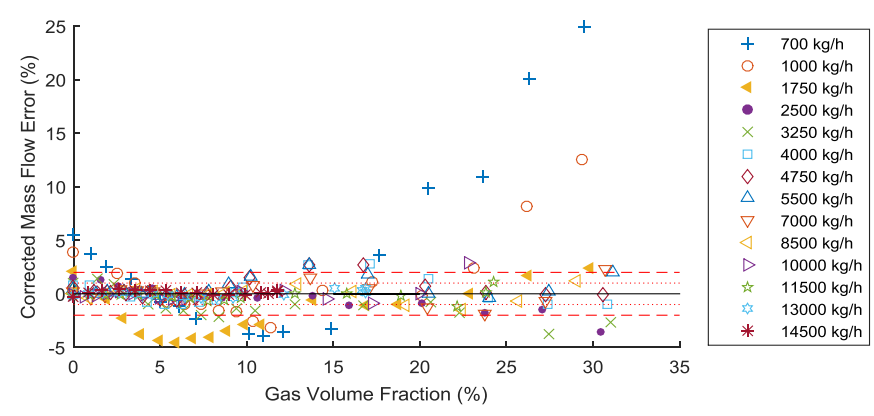

(c)

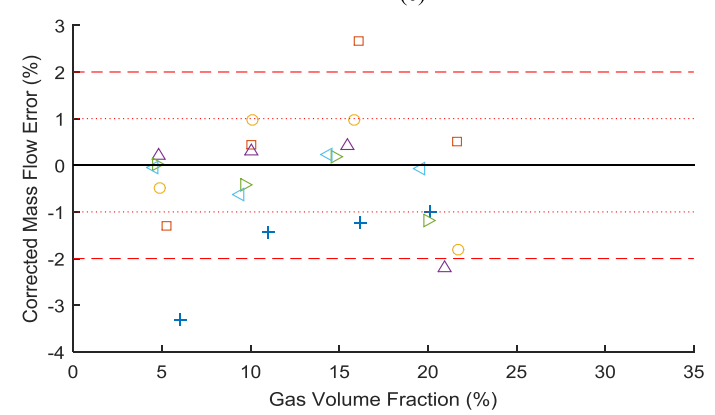

(d)

Fig. 18. Errors of the corrected liquid mass flowrate error from the GPs. (a) Errors of the corrected mass flowrate on the horizontal pipeline with training data set. (b) Errors of the corrected mass flowrate on the horizontal pipeline with test data set. (c) Errors of the corrected mass flowrate on the vertical pipeline with training data set. (d) Errors of the corrected mass flowrate on the vertical pipeline with test data set.

both the horizontal and vertical pipelines are around $\pm 30 \%$. The rest errors with the training data set are well within $\pm 10 \%$. The relative errors from the test data set are almost less than $\pm 10 \%$, except at the flowrate of $1000 \mathrm{~kg} / \mathrm{h}$ on the horizontal pipeline. This is probably due to the fact that the samples at $1000-\mathrm{kg} / \mathrm{h}$ flow rate are far away from the center vectors in the network.

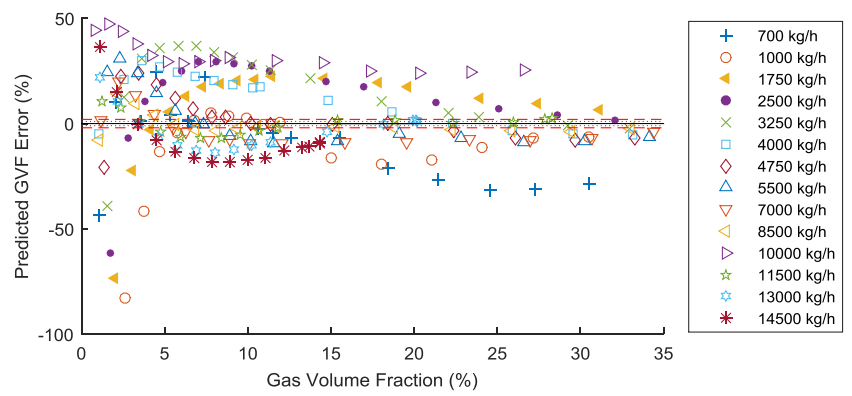

(a)

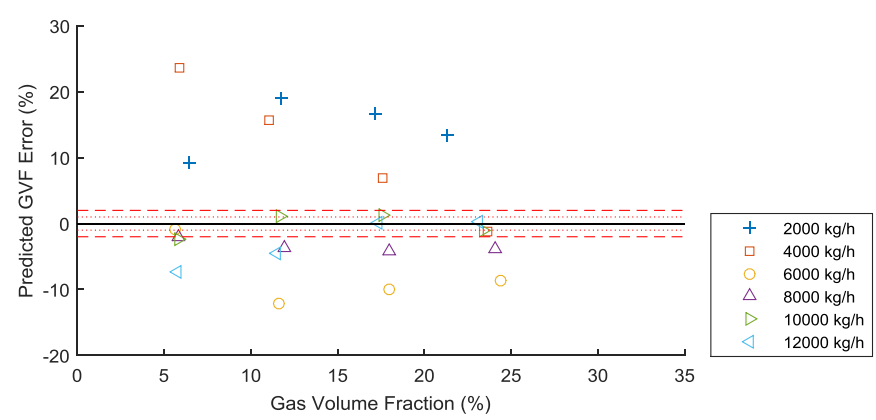

(b)
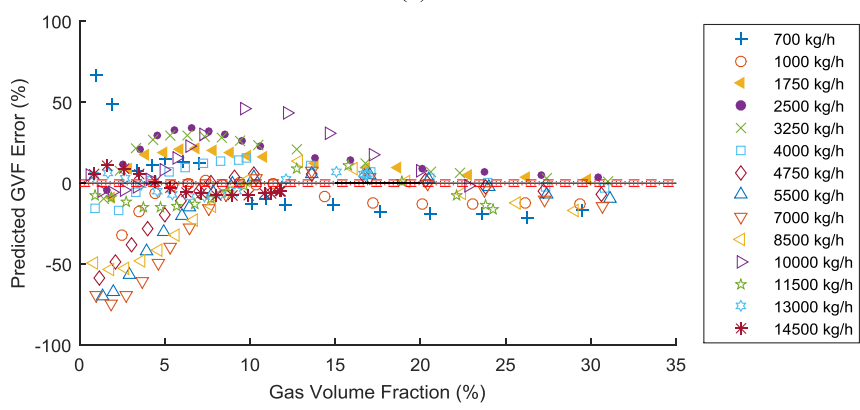

(c)

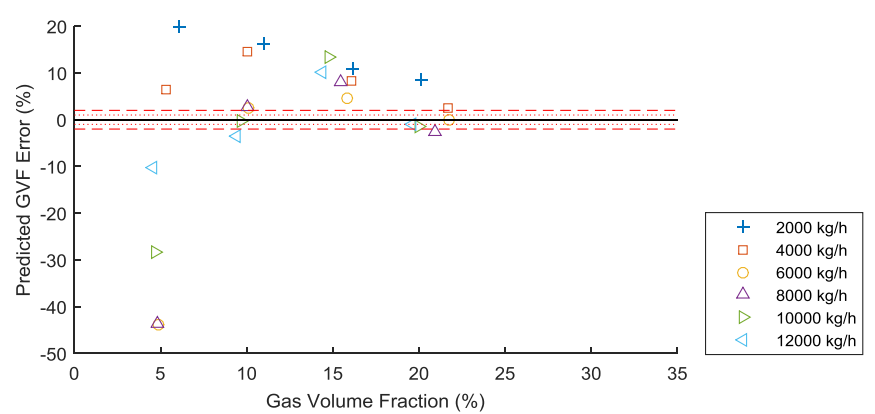

(d)

Fig. 19. Errors of the predicted gas volume fraction from the GPs. (a) Errors of the predicted gas volume fraction on the horizontal pipeline with training data set. (b) Errors of the predicted gas volume fraction on the horizontal pipeline with test data set. (c) Errors of the predicted gas volume fraction on the vertical pipeline with training data set. (d) Errors of the predicted gas volume fraction on the vertical pipeline with test data set.

\section{F. Performance of the SVM}

SVM models are also established for both installation conditions. An important difference between the SVM and ANN models is that the SVM leads to a unique deterministic model for each data set, while ANNs depend on a random 
TABLE III

NRMSE OF SVM WITH DIFFERENT KERNEL FUNCTIONS

\begin{tabular}{|c|c|c|c|c|c|c|c|c|}
\hline \multirow{2}{*}{ Model } & \multicolumn{4}{|c|}{ Data set 1 } & \multicolumn{4}{c|}{ Data set 2 } \\
\cline { 2 - 9 } & Linear & Polynomial & $\begin{array}{c}\text { Radial } \\
\text { basis } \\
\text { function }\end{array}$ & Sigmoid & Linear & Polynomial & $\begin{array}{c}\text { Radial } \\
\text { basis } \\
\text { function }\end{array}$ & Sigmoid \\
\hline H-L & $5.62 \%$ & $11.12 \%$ & $0.11 \%$ & 889.50 & $7.44 \%$ & $10.97 \%$ & $0.58 \%$ & 738.32 \\
\hline V-L & $6.32 \%$ & $10.33 \%$ & $0.10 \%$ & 911.90 & $9.39 \%$ & $11.42 \%$ & $0.57 \%$ & 777.32 \\
\hline H-G & $21.37 \%$ & $28.37 \%$ & $3.44 \%$ & 606.58 & $2.6 \%$ & $5.68 \%$ & $3.29 \%$ & 138.03 \\
\hline V-G & $27.27 \%$ & $34.08 \%$ & $2.16 \%$ & 683.13 & $3.71 \%$ & $6.78 \%$ & $3.2 \%$ & 171.56 \\
\hline
\end{tabular}

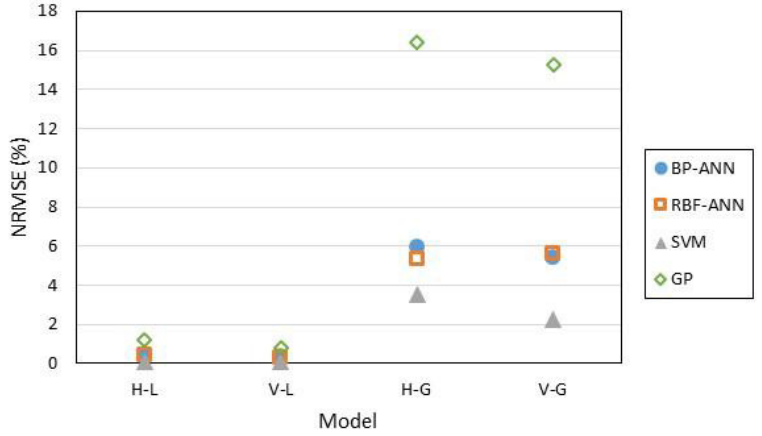

(a)

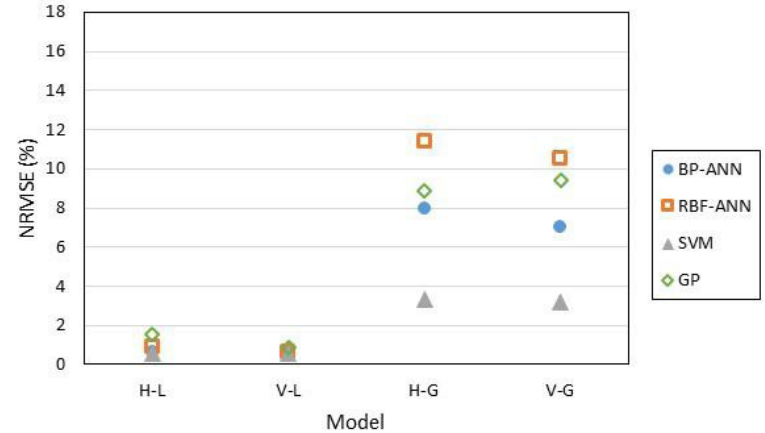

(b)

Fig. 20. Performance comparison between ANNs, SVMs, and GPs. (a) ANNs, SVMs, and GPs with training data set. (b) ANNs, SVMs, and GPs with test data set.

initial choice of synaptic weights and cannot produce the fixed results. Through a direct comparison of the performances of SVM between the four kinds of kernel function (Table III), we know that the SVM with RBF generates the smallest NRMSE among the four models.

From Fig. 16(a) and (c), the SVM model performs well to fit with training data and limit the relative errors on the horizontal and vertical pipelines to $\pm 1 \%$ or less, except some points at 700 and $1000 \mathrm{~kg} / \mathrm{h}$, which is a common problem for the ANN and SVM models. The generalization ability of the SVM model is proven, as shown in Fig. 16(b) and (d). Most errors from the SVM models with the test data are reduced to $\pm 1 \%$.

Fig. 17 shows that for gas volume fraction prediction, a less number of points from the SVM models have an error beyond $\pm 10 \%$ with the training data set. Since the kernel function used in the SVM models is RBF, the performance of the SVM models has the common problem with the RBF-ANN. The relative errors in the predicted gas volume fraction with the test data set at the flowrate of $1000 \mathrm{~kg} / \mathrm{h}$ are larger than other test data.

\section{G. Performance of the GP}

Four GP models are established in this paper for correcting the liquid mass flowrate and predicting the gas volume fraction, respectively, for the horizontal and vertical installations of Coriolis flowmeters. The parameters that were set in the GP algorithms include: a population size of 250 , a tournament size of 25 , an elitism of 0.7 , maximum number of genes allowed in an individual 6 , function set $\{\times,-,+, \tanh , \operatorname{mult} 3$, add 3$\}$, and terminal sets $\left\{x_{1}, x_{2}, x_{3}, x_{4}\right\}$ for models H-L and V-L and $\left\{x_{1}, x_{2}, x_{4}\right\}$ for models H-G and V-G.

The GP-based formulations for the four models are given in the following:

$$
\begin{aligned}
y_{H-L}= & 0.994 x_{2}-2633 x_{1}+4300 \tanh \left(x_{1}\right) \tanh \left(x_{3}\right) \\
& +13.2 x_{1} x_{4}+0.00571 x_{2} x_{3}-0.0995 x_{2} x_{3} \\
& \times \tanh \left(x_{1}\right)+62.4 \\
y_{V-L}= & x_{2}+57.6 x_{3}-0.161 x_{4}+29.8 x_{1} x_{4} \\
& +871 \tanh \left(x_{3}^{2} x_{4}\right) \tanh \left(x_{1}\right)-0.00913 x_{4}\left(x_{3}+x_{1} x_{4}\right) \\
& -0.122 x_{1} x_{2} x_{3}+32.5 \\
y_{H-G}= & 0.783 x_{1}+1.6 e^{-6} x_{2}+0.00278 x_{4}-0.114 x_{1} x_{4} \\
& +0.159 x_{1}^{2} x_{4}+6.82 e^{-5} x_{4}^{3}-0.0182 \\
y_{V-G}= & 1.01 x_{1}-5.49 e^{-7} x_{2}-0.0217 x_{4}-2.74 e^{-7} \tanh \left(x_{1}\right) \\
& -1.05 e^{-4} x_{1} x_{2}+2.74 e^{-6} x_{2} x_{4}+0.00253 x_{1} x_{4}^{2} \\
& -1.05 e^{-4} x_{1}^{2} x_{4}-2.74 e^{-7} x_{1} x_{2} x_{4}+0.00587
\end{aligned}
$$

The errors of the corrected mass flowrate on the training data set using GP are higher by $-15 \%$ and $25 \%$, respectively, under the horizontal and vertical installations [Fig. 18(a) and (c)], which results in larger errors on the test data set [Fig. 18(b) and (d)]. As can be seen that, larger errors normally occur at low flowrates, which indicate that the GP models are unable to approximate all the data.

As shown in Fig. 19, for the prediction of gas volume fraction, the outputs of GP models have large errors for low gas entrainment and low flowrates. The relative errors with test data reach $25 \%$ and $-50 \%$ on the horizontal and vertical pipes, respectively. 


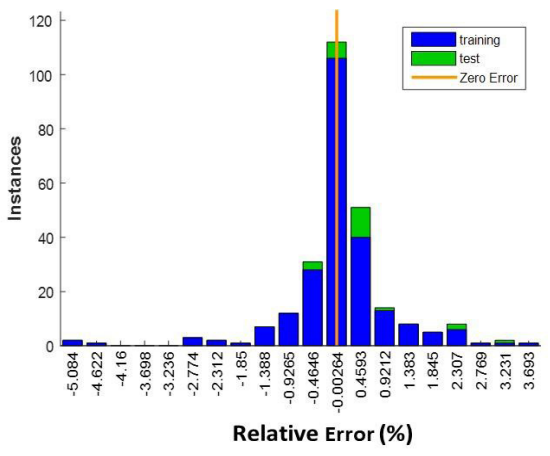

(1) BP-ANN: H-L

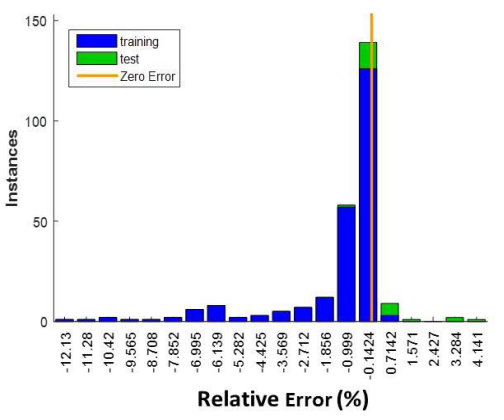

(3) RBF-ANN: H-L

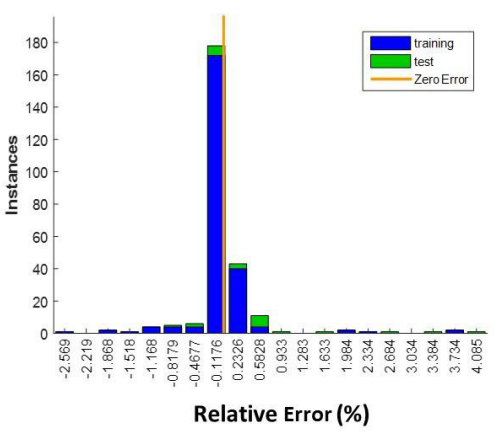

(5) SVM: H-L

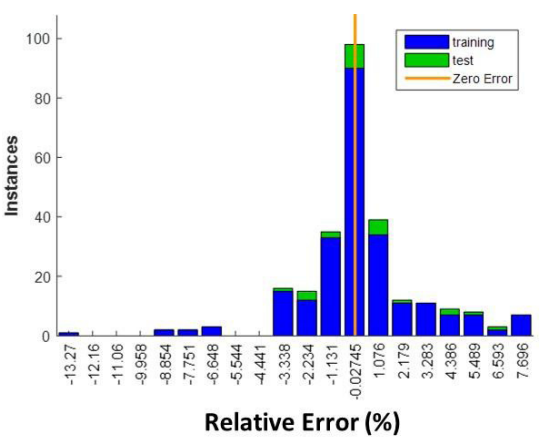

(7) GP: H-L

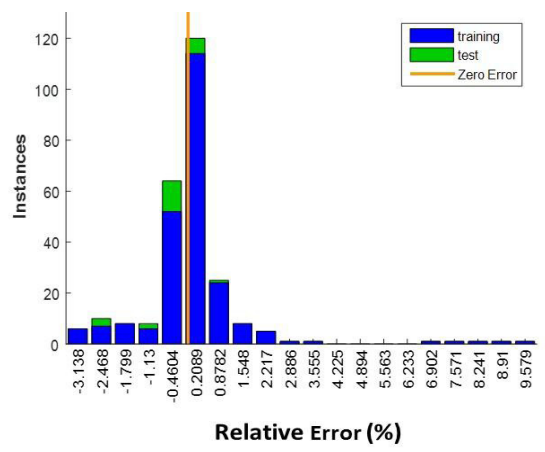

(2) BP-ANN: V-L

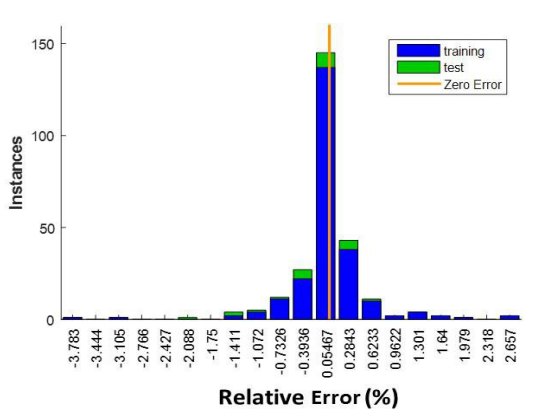

(4) RBF-ANN: V-L

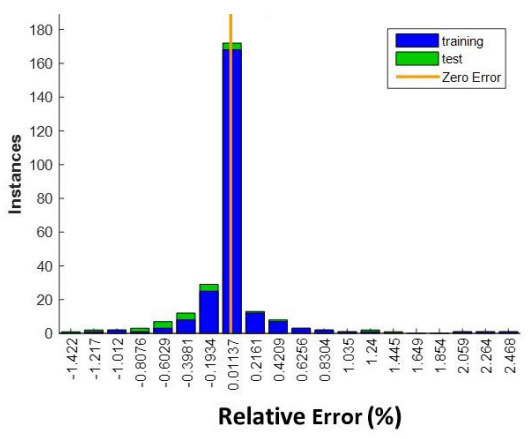

(6) SVM: V-L

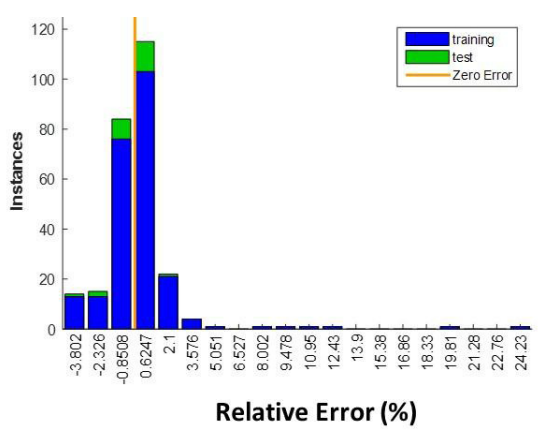

(8) GP: V-L

Fig. 21. Relative error histogram of ANNs, SVMs, and GPs for corrected liquid mass flowrate. (a) BP-ANN: H-L. (b) BP-ANN: V-L. (c) RBF-ANN: H-L. (d) RBF-ANN: V-L. (e) SVM: H-L. (f) SVM: V-L. (g) GP: H-L. (h) GP: V-L.

\section{H. Perforamce Comparison Between BP-ANN,} $R B F-A N N, S V M$, and GP

1) Robustness: In order to assess the robustness of the four kinds of models, the averaged NRMSE values are shown in Fig. 20. The models for liquid mass flowrate correction and gas volume fraction prediction, GP produces larger errors than the other three techniques. Both BP-ANN and RBF-ANN have a similar mean NRMSE with the training data set, while the SVM models yield less error. With the test data set, BP-ANN, RBF-ANN, and SVM methods perform similarly on Models 


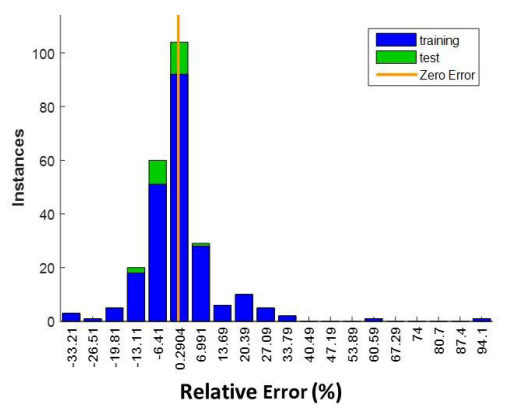

(1) BP-ANN: H-G

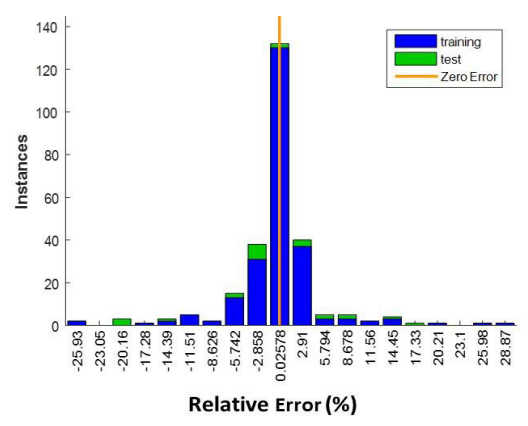

(3) RBF-ANN: H-G

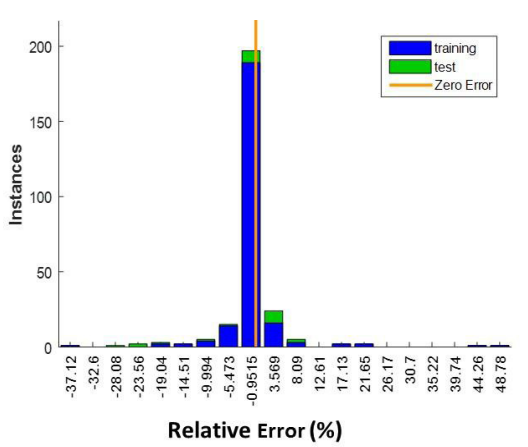

(5) SVM: H-G

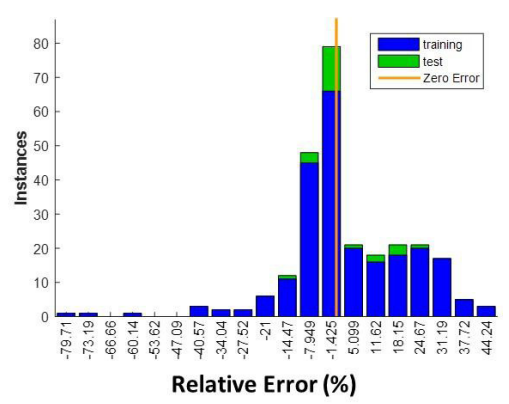

(7) GP: H-G

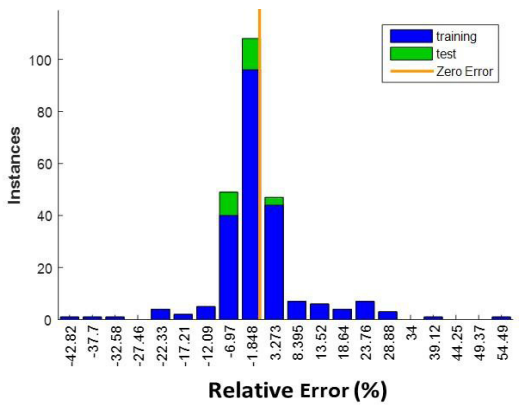

(2) BP-ANN: V-G

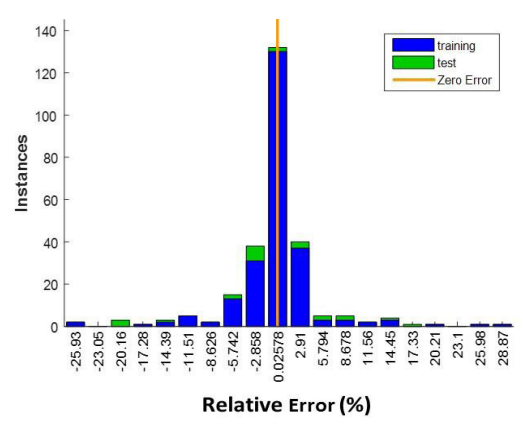

(4) RBF-ANN: V-G

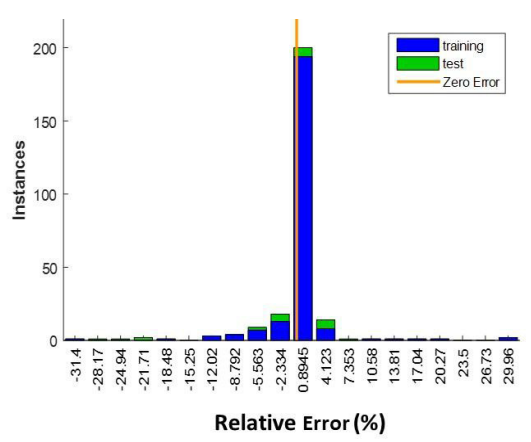

(6) SVM: V-G

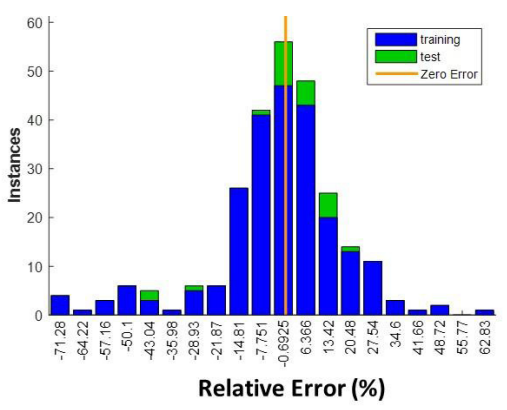

(8) GP: V-G

Fig. 22. Relative error histogram of ANNs, SVMs, and GPs for gas volume fraction prediction. (a) BP-ANN: H-G. (b) BP-ANN: V-G. (c) RBF-ANN: H-G. (d) RBF-ANN: V-G. (e) SVM: H-G. (f) SVM: V-G. (g) GP: H-G. (h) GP: V-G.

H-L and V-L. However, the SVM models are significantly better than the BP-ANN, RBF-ANN, and GP models for the prediction of gas volume fraction. Moreover, BP-ANN and RBF-ANN have uncertain parameters to optimize which could result in differences in performance. However, due to their fixed structure, the SVM models produce repeatable results all the time. This outcome suggests that the SVM models are superior to both ANN and GP models in terms of robustness.

2) Accuracy: Fig. 21 shows the relative error histograms of the ANNs, SVMs, and GPs for corrected liquid mass flowrate. It is clear that the error distributions of the GP and ANN models are much wider and dispersive than the SVM models. 
TABLE IV

Mean and Standard Deviation of the Relative Error Distribution for Liquid Mass Flowrate Correction

\begin{tabular}{|l|l|c|c|c|c|}
\hline Model & & BP-ANN & RBF-ANN & SVM & GP \\
\hline \multirow{2}{*}{ Model H-L } & Mean (\%) & 0.0823 & 1.2200 & 0.0585 & 0.2405 \\
\cline { 2 - 5 } & Standard deviation (\%) & 1.03 & 2.30 & 0.66 & 2.83 \\
\hline \multirow{2}{*}{ Model V-L } & Mean (\%) & 0.0548 & -0.0248 & 0.0008 & 0.1660 \\
\cline { 2 - 6 } & Standard deviation (\%) & 1.50 & 0.61 & 0.40 & 2.77 \\
\hline
\end{tabular}

TABLE V

Mean and Standard Deviation of the Relative Error Distribution for Gas Volume Fraction Prediction

\begin{tabular}{|l|l|c|c|c|c|}
\hline Model & & BP-ANN & RBF-ANN & SVM & GP \\
\hline \multirow{2}{*}{ Model H-G } & Mean (\%) & 0.17 & -0.26 & -0.25 & 3.15 \\
\cline { 2 - 5 } & Standard deviation (\%) & 11.88 & 6.02 & 6.95 & 17.70 \\
\hline \multirow{2}{*}{ Model V-G } & Mean (\%) & -0.18 & 0.50 & -0.38 & -1.99 \\
\cline { 2 - 6 } & Standard deviation (\%) & 9.70 & 4.70 & 5.57 & 20.62 \\
\hline
\end{tabular}

TABLE VI

ACCURACY COMPARISONS OF ANN, SVM, AND GP MODELS

\begin{tabular}{|l|c|c|c|c|c|c|}
\hline Model & \multicolumn{2}{|c|}{ H-L } & \multicolumn{2}{c|}{ V-L } & H-G & V-G \\
\hline Error limit & $\leq \pm 2 \%$ & $\leq \pm 1 \%$ & $\leq \pm 2 \%$ & $\leq \pm 1 \%$ & $\leq \pm 10 \%$ & $\leq \pm 10 \%$ \\
\hline BP-ANN & $91.95 \%$ & $80.08 \%$ & $89.66 \%$ & $79.69 \%$ & $79.31 \%$ & $86.21 \%$ \\
\hline RBF-ANN & $82.76 \%$ & $72.80 \%$ & $97.70 \%$ & $91.57 \%$ & $90.80 \%$ & $95.79 \%$ \\
\hline SVM & $96.93 \%$ & $93.49 \%$ & $98.85 \%$ & $96.17 \%$ & $93.10 \%$ & $94.25 \%$ \\
\hline GP & $68.20 \%$ & $54.41 \%$ & $83.14 \%$ & $67.05 \%$ & $55.56 \%$ & $54.79 \%$ \\
\hline
\end{tabular}

Through comparing the mean value and standard deviation of the errors between the eight error distributions (Table IV), we can see that the SVM models with the lowest mean value and standard deviation outperform the BP-ANN, RBF-ANN, and GP models for liquid mass flowrate measurement on both the horizontal and vertical pipelines. Moreover, the data driven models (a mean value of $0.0008 \%$ and a standard deviation of $0.40 \%$ ) on the vertical pipeline perform better than those on the horizontal pipeline (a mean value of $0.0585 \%$ and a standard deviation of $0.66 \%$ ).

Fig. 22 shows the relative error histograms of the four types of models for gas volume fraction prediction. GP models have a larger range of errors than all other models. The error distribution of the SVM model is much narrower than the ANN models for the measurement of gas volume fraction. It can be seen that most errors of the SVM models are concentrated around zero line. Table $\mathrm{V}$ shows that the standard deviations of the SVM and RBF-ANN models are smaller than that of the BP-ANN and GP models on both the horizontal and vertical pipelines.

In order to assess the accuracy of the ANN, SVM, and GP models, the percentage of experimental data for each model that can achieve the accuracy of $\pm 2 \%$ and $\pm 1 \%$, respectively, for liquid mass flowrate measurement and $\pm 10 \%$ for gas volume fraction prediction is calculated and summarized in Table VI. For liquid mass flowrate measurement with the SVM models, $93.49 \%$ of the experimental data yield a relative error less than $\pm 1 \%$ on the horizontal pipeline, while $96.17 \%$ of the results are within $\pm 1 \%$ on the vertical installation. The SVM models predict the gas volume fraction with a relative error less than $10 \%$ for $93.10 \%$ and $94.25 \%$ of the test conditions on the horizontal and vertical installations, respectively. Therefore, the SVM models perform significantly better than the BP-ANN, RBF-ANN, and GP models for twophase flow measurement in terms of robustness and accuracy.

\section{CONCLUSION}

In this paper, experimental and analytical investigations have been carried out to assess the performance of BP-ANN, RBF-ANN, SVM, and GP for gas-liquid two-phase flow measurement using Coriolis flowmeters. The results presented have suggested that the SVM models are superior to the two ANN models and the GP models for two-phase flow measurement in terms of robustness and accuracy. The SVM models perform well consistently, while the performance of ANN and GP models depends on the user-defined parameters. For liquid mass flowrate measurement, the SVM models outperform the BP-ANN, RBF-ANN, and GP on both the horizontal and vertical pipelines and the most corrected errors $(>93 \%)$ are within $\pm 1 \%$. For the gas volume fraction prediction, the RBF-ANN and SVM models yield most relative errors (>90\%) less than $\pm 10 \%$ and outperform the BP-ANN and GP. It must be stressed that the significantly reduced errors in 
mass flowrate measurement from the Coriolis mass flowmeters and gas volume fraction prediction are achieved by using the existing data from the Coriolis flowmeters and a simple DP transducer without the use of any other devices. SVM has consistently outperformed ANN and GP in the correction of liquid mass flow errors and prediction of gas volume fraction. This outcome has effectively extended the applicability of Coriolis mass flowmeters to liquid flow measurement with a significant volume of entrained gas. In the future work, the data driven models will be extended for the measurement of other liquids with different viscosities under two-phase or multiphase flow conditions.

\section{REFERENCES}

[1] Y. Li, J. Wang, and Y. Geng, "Study on wet gas online flow rate measurement based on dual slotted orifice plate," Flow Meas. Instrum., vol. 20, nos. 4-5, pp. 168-173, Aug. 2009.

[2] J. L. G. Oliveira, J. C. Passos, R. Verschaeren, and C. van der Geld, "Mass flow rate measurements in gas-liquid flows by means of a Venturi or orifice plate coupled to a void fraction sensor," Experim. Thermal Fluid Sci., vol. 33, no. 2, pp. 253-260, Jan. 2009.

[3] Z. Sun, "Mass flow measurement of gas-liquid bubble flow with the combined use of a Venturi tube and a vortex flowmeter," Meas. Sci. Technol., vol. 21, no. 5, pp. 1-7, Mar. 2010.

[4] Y. Xu, H. Wang, Z. Cui, F. Dong, and Y. Yan, "Separation of gas-liquid two-phase flow through independent component analysis," IEEE Trans. Instrum. Meas., vol. 59, no. 5, pp. 1294-1302, May 2010.

[5] L. Wang, Z. Yao, B. Wang, H. Ji, and H. Li, "Flow pattern identification of gas-liquid two-phase flow based on capacitively coupled contactless conductivity detection," IEEE Trans. Instrum. Meas., vol. 61, no. 5, pp. 1466-1475, May 2012.

[6] F. Dong, Y. Xu, L. Hua, and H. Wang, "Two methods for measurement of gas-liquid flows in vertical upward pipe using dual-plane ERT system," IEEE Trans. Instrum. Meas., vol. 55, no. 5, pp. 1576-1586, Oct. 2006.

[7] L. Xu, W. Zhou, X. Li, and S. Tang, "Wet gas metering using a revised Venturi meter and soft-computing approximation techniques," IEEE Trans. Instrum. Meas., vol. 60, no. 3, pp. 947-956, Mar. 2011.

[8] X. Li, X. Huang, B. Wang, and H. Li, "A new method for the online voidage measurement of the gas-oil two-phase flow," IEEE Trans. Instrum. Meas., vol. 58, no. 5, pp. 1571-1577, May 2009.

[9] H. Ji, J. Long, Y. Fu, Z. Huang, B. Wang, and H. Li, "Flow pattern identification based on EMD and LS-SVM for gas-liquid two-phase flow in a minichannel," IEEE Trans. Instrum. Meas., vol. 60, no. 5, pp. 1917-1924, May 2011.

[10] M. G. De Giorgi, A. Ficarella, and A. Lay-Ekuakille, "Cavitation regime detection by LS-SVM and ANN with wavelet decomposition based on pressure sensor signals," IEEE Sensors J., vol. 15, no. 10, pp. 5701-5708, Oct. 2015.

[11] T. Wang and R. Baker, "Coriolis flowmeters: A review of developments over the past 20 years, and an assessment of the state of the art and likely future directions," Flow Meas. Instrum., vol. 40, pp. 99-123, Sep. 2014.

[12] J. Hemp and G. Sultan, "On the theory and performance of coriolis mass flowmeters," in Proc. Int. Conf. Mass Flow Meas., 1989, pp. 1-38.

[13] R. P. Liu, M. J. Fuent, M. P. Henry, and M. D. Duta, "A neural network to correct mass flow errors caused by two-phase flow in a digital coriolis mass flowmeter," Flow Meas. Instrum., vol. 12, no. 1, pp. 53-63, Mar. 2001.

[14] B. Safarinejadian, M. A. Tajeddini, and L. Mahmoodi, "A new fuzzy based method for error correction of coriolis mass flow meter in presence of two-phase fluid," in Proc. Int. Conf. Artif. Intell. Image Process., 2012, pp. 192-196.

[15] V. A. Lari and F. Shabaninia, "Error correction of a coriolis mass flow meter in two-phase flow measurment using neuro-fuzzy," in Proc. 16th CSI Int. Symp. Artif. Intell. Signal Process., May 2012, pp. 611-616.

[16] Q. L. Hou, K. J. Xu, M. Fang, Y. Shi, B. B. Tao, and R. W. Jiang, "Gas-liquid two-phase flow correction method for digital CMF," IEEE Trans. Instrum. Meas., vol. 63, no. 10, pp. 2396-2404, Mar. 2014.
[17] L. Xing et al., "A combination method for metering gas-liquid twophase flows of low liquid loading applying ultrasonic and coriolis flowmeters," Flow Meas. Instrum., vol. 37, pp. 135-143, Jan. 2014.

[18] L. Ma, H. Zhang, H. Zhou, and Q. He, "Mass flow measurement of oilwater two-phase flow based on coriolis flow meter and SVM," J. Chem. Eng. Chin. Univ., vol. 21, no. 2, pp. 201-205, Apr. 2007.

[19] R. May, H. Maier, G. C. Dandy, and T. Fernando, "Non-linear variable selection for artificial neural networks using partial mutual information," Environ. Model. Softw., vol. 23, nos. 10-11, pp. 1312-1326, 2008.

[20] S. Galelli, G. B. Humphrey, H. R. Maier, A. Castelletti, G. C. Dandy, and M. S. Gibbs, "An evaluation framework for input variable selection algorithms for environmental data-driven models," Environ. Model. Softw., vol. 62, pp. 33-51, Dec. 2014.

[21] L. Wang, J. Liu, Y. Yan, X. Wang, and T. Wang, "Gas-liquid twophase flow measurement using coriolis flowmeters incorporating neural networks," in Proc. IEEE Int. Instrum. Meas. Technol. Conf., Taipei, Taiwan, May 2016, pp. 747-751.

[22] H. A. Farfani, F. Behnamfar, and A. Fathollahi, "Dynamic analysis of soil-structure interaction using the neural networks and the support vector machines," Expert Syst. Appl., vol. 42, no. 22, pp. 8971-8981, Dec. 2015.

[23] S. Belaid and A. Mellit, "Prediction of daily and mean monthly global solar radiation using support vector machine in an arid climate," Energ. Convers. Manage., vol. 118, pp. 105-118, Jun. 2016.

[24] M. A. M. Ramli, S. Twaha, and Y. A. Al-Turki, "Investigating the performance of support vector machine and artificial neural networks in predicting solar radiation on a tilted surface: Saudi Arabia case study," Energ. Convers. Manage., vol. 105, pp. 442-452, Nov. 2015.

[25] F. Chen, H. Li, Z. Xu, S. Hou, and D. Yang, "User-friendly optimization approach of fed-batch fermentation conditions for the production of iturin a using artificial neural networks and support vector machine," Electron. J. Biotechnol., vol. 18, no. 4, pp. 273-280, Jul. 2015.

[26] A. H. Gandomi and A. H. Alavi, "A new multi-gene genetic programming approach to nonlinear system modeling. Part I, materials and structural engineering problems," Neural Comput. Appl., vol. 21, no. 1, pp. 171-187, Feb. 2012.

[27] C. Cortes and V. Vapnik, "Support-vector networks," Mach. Learn., vol. 20, no. 3, pp. 273-297, 1995.

[28] H. Drucker, C. Burges, L. Kaufman, A. Smola, and V. Vapnik, "Support vector regression machines," Neural Inf. Process. Syst., vol. 9, pp. 155-161, Apr. 1997.

[29] J. Koza, Genetic Programming: On the Programming of Computers by Means of Natural Evolution. Cambridge, MA, USA: MIT Press, 1992.

[30] J. Madár, J. Abonyi, and F. Szeifert, "Genetic programming for the identification of nonlinear input-output models," Ind. Eng. Chem. Res., vol. 44, no. 9, pp. 3178-3186, Mar. 2005.

[31] D. P. Searson, "GPTIPS 2: An open-source software platform for symbolic data mining," in Handbook of Genetic Programming Applications. New York, NY, USA: Springer, 2015, ch. 22.

[32] J. W. Kunze, R. Storm, and T. Wang, "Coriolis mass flow measurement with entrained gas," in Proc. ITG/GMA Symp. Sensors Meas. Syst., Jun. 2014, pp. 1-6.

[33] G. J. Bowden, H. R. Maier, and G. C. Dandy, "Input determination for neural network models in water resources applications. Part 2. Case study: Forecasting salinity in a river," J. Hydrol., vol. 301, nos. 1-4, pp. 93-107, Jan. 2005.

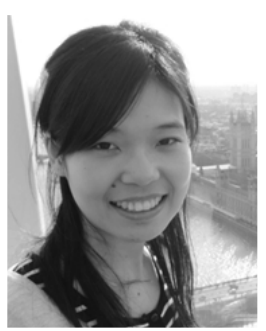

Lijuan Wang (S'13) received the B.Eng. degree in computer science and technology from Qiqihar University, Qiqihar, China, in 2010, and the Ph.D. degree in measurement and automation from North China Electric Power University, Beijing, China, in 2015. She is currently pursuing the Ph.D. degree in instrumentation and measurement with the University of Kent, Canterbury, U.K.

Her current research interests include electrostatic sensing, flow measurement, and digital signal processing. 


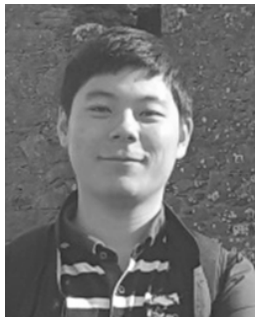

Jinyu Liu received the B.Eng. degrees in electrical engineering and automation from Tianjin University, Tianjin, China, and in electronic and computer systems from the University of Kent, Canterbury, U.K., in 2012, and the M.Sc. degree in energy and sustainability with electrical power engineering from the University of Southampton, Southampton, U.K., in 2013. He is currently pursuing the Ph.D. degree in instrumentation and measurement with the University of Kent.

His current research interests include flow rig construction, flow measurement, and digital signal processing.

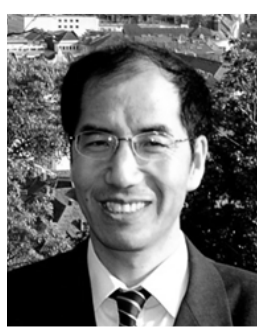

Yong Yan (M'04-SM'04-F'11) received the B.Eng. and M.Sc. degrees in instrumentation and control engineering from Tsinghua University, Beijing, China, in 1985 and 1988, respectively, and the Ph.D. degree in flow measurement and instrumentation from the University of Teesside, Middlesbrough, U.K., in 1992.

He was an Assistant Lecturer with Tsinghua University in 1988. In 1989, he joined as a Research Assistant with the University of Teesside. He was a Lecturer with the University of Teesside from 1993 to 1996, and a Senior Lecturer, a Reader, and a Professor with the University of Greenwich, Chatham Maritime, U.K., from 1996 to 2004. He is currently a Professor of Electronic Instrumentation, the Head of Instrumentation, Control and Embedded Systems Research Group, and the Director of Research with the School of Engineering and Digital Arts, University of Kent, Canterbury, U.K. His current research interests include multiphase flow measurement, combustion instrumentation, and monitoring and optimization of industrial processes.

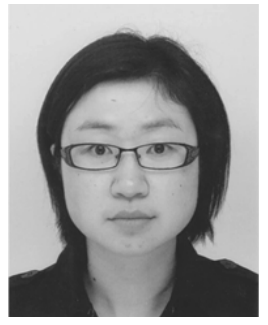

Xue Wang received the B.Sc. degree in mathematical statistics from Northwest University, Xi'an, China, in 1996, the M.Sc. degree in statistics from Northwestern Polytechnic University, Xi'an, in 1999, and the Ph.D. degree in statistics from The University of Nottingham, Nottingham, U.K., in 2006.

She was a Post-Doctoral Research Assistant with the Statistics Group, University of Bristol, Bristol, U.K., from 2005 to 2006. She is currently a Lecturer with University of Kent, Canterbury, U.K. Her current research interests include bayesian nonparametrics, wavelet and multiscale methods, and statistical signal processing.

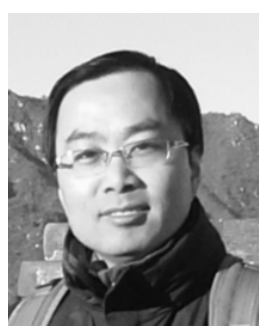

Tao Wang received the B.Eng. and M.Sc. degrees in aerospace manufacturing engineering from Northwestern Polytechnic University, Xi'an, China, in 1996 and 1999, respectively, and the Ph.D. degree in engineering from the University of Cambridge, Cambridge, U.K., in 2003.

He was a Research Associate with the University of Cambridge from 2002 to 2004 and a Lecturer with Brunel University, London, U.K., from 2007 to 2008. He is currently the Research and Metrology Manager with KROHNE Ltd., Wellingborough, U.K., and a Senior Visiting Research Fellow with the School of Engineering and Digital Arts, University of Kent, Canterbury, U.K. His current research interests include flow measurement and instrumentation, Coriolis flowmeter technology, numerical simulation, and manufacturing engineering. 